Ann. Abeille, Ig66, $9(\mathrm{I}), 5-36$.

\title{
L'INFLUENCE DE LA REINE D'ABEILLE (APIS MELLIFICA L.) SUR LE TAUX DE MORTALITÉ DES OUVRIÈRES ACCOMPAGNATRICES
}

\author{
B. ROGER et Tanine PAIN \\ Station de Recherches sur l'Abeille et les Insecles sociaux, \\ 91 - Bures-sur-Yoette
}

SOMMAIRE

Dans le cadre des recherches sur les interactions entre reine et ouvrières d'abeilles, les auteurs étudient l'influence de reines vivantes, vierges ou fécondes, jeunes ou vieilles, de reines mortes, de broyats céphaliques royaux et de la phérormone I de synthèse, sur la mortalité des ouvrières accompagnatrices.

L'analyse des courbes de mortalité montre que la présence des reines vivantes abaisse le taux de mortalité de petits groupes de 50 ou 100 ouvrières, mais à des degrés divers. Ce sont les reines vierges vieillies en étuve qui réduisent le plus cette mortalité. Un apport de pollen ne masque pas l'infuence des reines.

Des cadavres de reines fécondes attractives ont également une action sur la réduction du taux de mortalité. Cette action est de même intensité que celle de jeunes reines vierges vivantes.

L'influence des reines ne se fait sentir qu'après plus de 15 jours d'encagement des ouvrières auprès des reines.

La phérormone I de synthèse, non attractive, réduit, mais très faiblement, la mortalité des ouvrières.

On émet l'hypothèse d'une action phérormonale, peut-être liée à l'odeur royale, mais d'autres actions pourraient aussi entrer en jeu.

\section{INTRODUCTION}

Le maintien d'abeilles en captivité a permis à un certain nombre d'auteurs d'étudier la durée de vie d'ouvrières orphelines, mais les études relatives à la mortalité d'ouvrières encagées en présence d'une reine n'ont pas suscité autant d'intérêt. C'est pourquoi il nous a paru nécessaire d'entreprendre ce travail en tenant compte des données actuelles concernant l'influence de la reine sur les ouvrières. Ces dernières, en effet, subissent de la part de la reine plusieurs influences. PAIN (Ig6r) ainsi que BUTLER (I96I) ont montré qu'à son contact les ouvrières ne construisent pas de 
cellules royales, mais accélèrent, par contre, la construction de cellules d'ouvrières (Chauvin, I) archen, PAin, I96r). I)e plus, les ouvrières sont attirées par son odeur et elles restent stériles (PAIN, I955). Ces différents processus (inhibition et attraction) sont déclenchés par l'existence d'une phérormone rovale, contenue dans les glandes mandibulaires.

En tenant compte de ces données, nous nous sommes demandlé si la reine pouvait exercer encore d'autres influences. Nous avons pensé à son action possible sur le taux de mortalité d'ouvrières encagées. J'insiste sur cette idée que s'il nous a paru utile de revenir une nouvelle fois sur un sujet déjà très étudié, c'est que dans le domaine des mortalités, les rapports entre reine et ouvrières n'ont pas été soupçonnés et qu'il importait d'essayer de les mettre en évilence.

Les facteurs influençant la durée de vie des abeilles ont fait l'objet de très nombreux travaux, depuis le début de ce siècle. I,es recherches que nous passerons maintenant en revue ont porté, soit sur la durée de vie d'abeilles appartenant à des colonies en liberté, soit sur celle d'abeilles maintenues dans des cagettes expérimentales dans des conditions contrôlées.

\section{Io Durée de rie des abeilles enlliberté}

La durée de vie des abeilles dans la ruche est différente selon les saisons. F,n marquant les abeilles et en les prélevant à des intervalles cle temps réguliers, on a pu mettre en évidence, dans les pays tempérés aux saisons bien définies, que les abeilles d'été sont des abeilles à durée de vie courte. lilles vivent de 3 à 8 semaines. I es abeilles d'hiver sont des abeilles à durée de vie longue. Filles vivent de 6 à 8 mois (ANDERSON, I93I ; BuTTEI-REEIEN, I9I5 ; DEMUTH, I926 ; EVWNIUS, I937 ; KEIILR, I920; Maurizio, I946, I954, I959; Milum, I93 ; Nickil et Armbrustis, I937, I938; Philitips, I922, I939; PIXEI-GOODRICH, I920; RiI.EY, I895; RÖSCH, I925 ; SENX, I92I).

Selon Maurizio (I954), des abeilles marquées introduites en automme dans les colonies d'essais, se retrouvaient âgées de 2 I 5 à 233 jours en avril et mai.

Selon FREE et SPENCER-BOOTH (I959), la durée de vie d'ouvrières écloses en mars est en moyenne de 35 jours, alors que celle d'ouvrières écloses en juin n'est plus que de 28 jours. La durée de vie des abeilles d'été a donc, en trois mois, diminué d'une semaine. Les auteurs signalent aussi des variations dans la durée de vie des ouvrières en provenance de colonies différentes.

De nombreuses abeilles écloses en aôtit peuvent hiverner ; leur taux de mortalité, relevé au printemps suivant, sera cependant plus élevé que celui d'abeilles écloses en octobre. Pour expliquer cette diminution de la durée de vie des abeilles d'été, certains auteurs (PHILI,IS, I922, I928; Schmin'T, I923) ont supposé qu'elle était fonction de l'activité des butineuses. Il semble cependant que ce facteur ne soit pas très important. Ainsi Rocksster (I950) exposant des abeilles en cage à une lumière continue, constata que leur longévité éta1t de 2 à 3 semaines plus longue que celles des abeilles témoins maintenues dans des conditions normales d'éclairement et de vol. Cependant, Husing (I953) affirme qu'il n'est pas encore possible de conclure que l'activité de vol soit absolument sans action. Par contre, MaUERmaYer (I954) ne constate que très peu de différence, quant à la valeur moyenne de la durée de vie maximum, entre abeilles de colonies au travail tout le jour, et abeilles ne travaillant 
que la moitié de celui-ci ; dans ces expériences, la température et 1'humidité relative ne paraissent pas non plus avoir d'influence.

Par ailleurs, un facteur racial vient compliquer les données relatives à la longévité en fonction de l'activité. Ainsi, EL-DEEB (I952) a montré qu'au cours de l'été, les ouvrières de race Italienne vivent moins longtemps que les ouvrières des races Caucasienne et Carniolienne, à cause de leur activité intense d'élevage et de butinage. Mais, au cours de l'hiver, ce sont les abeilles de race Italienne qui ont la durée de vie la plus longue. HASSANEIN et EL BANBy (I960) indiquent que les ouvrières de race Caucasienne, au cours de certaines floraisons, présentent presque toujours une durée de vie plus longue que celle des autres races.

La réduction de la durée de vie des abeilles d'été est surtout fonction des conditions d'élevage et de la présence de couvain. Selon Mavrizio (I954), dans la colonie possédant du couvain, les abeilles atteignent l'âge de 60 jours au maximum, alors que dans la colonie sans couvain et par conséquent orpheline, la longévité varie entre 167 et I 88 jours. Ainsi, en été, 1'absence de couvain dans une colonie augmente la longévité des abeilles. En tenant compte de travaux plus anciens (EvEnIUs, I937 ; FARRAR, I936 ; GONTARSKI, I950, I954; KOEHLER, I92I ; KRATKY, I93I ; LOTMAR, I939; MoskovljEvic, I939), l'auteur établit une relation entre la longévité et l'état physiologique des abeilles.

Maurizio (1954) résume ainsi les faits : "Dans la colonie possédant du couvain, les glandes nourricières développées décrurent après 30 à 40 jours au plus; le corps adipeux n'atteignit pas le maximum de son développement; les ovaires ne se développèrent pas. IJans la colonie sans couvain, les glandes nourricières, le corps adipeux et les ovaires parvinrent à l'apogée de leur développement après 4 à 6 semaines... " Si l'on supprime l'élevage du couvain, des "abeilles d'hiver" physiologiques apparaissent dans la colonie pendant la saison active; elles diffèrent cependant des véritables abeilles d'hiver par le non développement de leurs ovaires.

VOOGD (I959) trouva cependant que le développement ovarien augmente au cours de 1'été, en même temps que l'accroissement du nid à couvain.

Dans nos régions, si la présence de couvain diminue la durée de vie des abeilles d'été, aux Indes, la présence de couvain réduit la durée de vie des abeilles d'hiver et de printemps (KAPI ${ }_{4}$ I957), ces deux saisons étant celles où l'activité d'élevage est la plus grande.

\section{$2^{\circ}$ Durée de vie des abeilles claustrées}

La durée de vie des abeilles encagées a spécialement retenu notre attention. Les facteurs qui l'influencent sont nombreux.

a) Importance du groupement.

Grasse et Chauvin (I944) ont montré que les Insectes sociaux et spécialement les Abeilles ne supportent pas 1'isolement. En effet, ils meurent rapidement quand on les soustrait à l'influence du groupe. Étudiant la durée de vie moyenne d'ouvrières isolées, Chauvin (I952) note qu'elle n'est que de 6 jours. Il indique qu'il n'existe pas de variations très larges de part et d'autre de ce chiffre au cours de l'année. La survie est améliorée dès que les abeilles sont par groupes de 5 ou ro. Le chiffre de 30 abeilles paraît convenir pour les études sur la durée de vie des abeilles encagées 
b) Infuence de la saison et de l'âge des abeilles.

Extraite de la ruche pour être claustrée au laboratoire, sans liberté de vol l'abeille subit encore 1'influence de la saison.

BerTholf (I942) étudia, à $28^{\circ} \mathrm{C}$ et 45 p. Ioo d'humidité relative, la longévité de groupes d'importance variable d'abeilles d'âges connu et inconnu. Elles étaient nourries uniquement avec un sirop de sucre concentré. La durée moyenne de vie d'abeilles gardiennes varia au cours de II mois d'observation de $9, \mathrm{I} 3 \pm 0,580$ à 22,9 $\pm \mathrm{I}, 065$ jours, avec une moyenne de $\mathrm{r} 6,24 \pm 0,20$ pour le lot entier. Les abeilles encagées l'hiver montrèrent une légère augmentation de la longévité par rapport aux abeilles encagées l'été. La longévité des abeilles encagées au printemps et à l'automne se place entre celle des deux autres. Avec des abeilles d'âges variés allant de $\mathrm{I} 2 \mathrm{~h}$ à 80 jours au début de l'expérience, l'auteur ne constata pas de diminution nette de la longévité jusqu'au $30^{\circ}$ jour d'encagement. Ensuite, il nota une diminution graduelle jusqu'au $90^{\mathrm{e}}$ jour de captivité.

Si nous insistons sur les résultats de BERTHOLF, c'est parce que dans les expériences qu'il a décrites, certains groupes de jeunes abeilles possédaient une reine. D'après l'auteur celle-ci n'agirait pas sur la longévité des ouvrières ; nous relevons pourtant dans son travail (p. 889) que la longévité moyenne d'un groupe de 50 jeunes abeilles avec reine est de 46,72 jours ; celle du groupe sans reine est de 33,95 jours soit un écart de 12,7 jours en faveur des ouvrières non orphelines.

Maurizio (I946) précise qu'én été la durée de vie moyenne d'abeilles claustrées est de 20 jours, en hiver de 30 jours. De jeunes abeilles écloses en mai, montrent en captivité une durée de vie moyenne de 24 jours. Puis la durée de vie diminue pour les abeilles écloses en juin et juillet. Elle remonte avec les éclosions d'aôt et septembre. Les abeilles d'âge indéterminé, prélevées également en mai dans une même ruche, présentent une durée de vie moyenne de $2 I, 8$ jours. Elle est légèrement plus courte que celle des jeunes abeilles observées le même mois. La durée de vie de ces abeilles plus âgées va ensuite augmenter, avec des variations, jusqu'au mois d'avril de l'année suivante.

\section{c) Importance de l'eau.}

L'abeille encagée ne peut vivre sans recevoir une certaine quantité d'eau. Woonrow (I935) a montré que lorsque les abeilles reçoivent une nourriture liquide telle qu'une solution de sucre à 50 p. Ioo, elles vivent plus longtemps dans une atmosphère peu humide. Les abeilles ne pourraient, en présence de fortes hygrométries, éliminer l'eau en excès accumulée dans le contenu de l'ampoule rectale.

L,e même auteur (I.94I) montra que des abeilles alimentées de candi vivent plus longtemps lorsqu'elles reçoivent de l'eau. Mais lorsque l'humidité relative est voisine de $100 \mathrm{p}$. $I O 0$,à $22^{\circ} \mathrm{C}$, les ouvrières vivent environ le même temps avec du candi qu'elles soient en 1'absence ou en présence d'eau. Toujours selon Woodrow, !la durée de vie des reines est aussi améliorée quand elles ont de l'eau à leur disposition. L'humidité ambiante a sur elles peu d'effet.

Maurizio (I945) montra par ailleurs que des abeilles assoiffées vivent 3 fois moins longtemps que les autres. Nous avons constaté (PAIN, non publié) que des abeilles jeunes alimentées de candi ne peuvent vivre sans eau à $30^{\circ} \mathrm{C}$ que pendant 
8 à ro jours. Ians des conditions identiques, la mortalité est encore plus rapide chez des abeilles d'âge indéterminé.

\section{d) Influence de la température.}

Dans la colonie normale, la température est réglée par une vaste population et, de ce fait, elle est maintent1e à une valeur relativement constante. Il n'en va pas de même pour les abeilles encagées par petits groupes, ceux-ci étant beaucoup plus exposés aux variations de la température ambiante.

La plupart des auteurs ont travaillé avec les abeilles claustrées à 30 ou $34^{\circ} \mathrm{C}$. Cependant, KELLFR-KITZINGER (I935) observa une mortalité plus faible chez des abeilles encagées à $23^{\circ} \mathrm{C}$.

MELAMPY et MCGREGOR (I939) trouvèrent par contre, tout en faisant varier l'hygrométrie, que les abeilles vivent plus longtemps à $35^{\circ}$ qu'à $25^{\circ}$.

Les expériences de WoOdrow (I94I) déjà citées, précisent que les basses températures $\left(20-24^{\circ} \mathrm{C}\right)$ sont plus favorables que les hautes $\left(37^{\circ} \mathrm{C}\right)$ lorsque les ouvrières sont alimentées uniquement de candi. Les hautes températures sont plus favorables lorsque les ouvrières sont alimentées de candi et reçoivent de l'eau.

Comme le souligne PHirlips (I946), le problème des effets de la température sur la durée de vie des abeilles n'est pas simple; elle affecte tous les processus vitaux des ouvrières.

MaURIzro (I946) expérimenta avec des abeilles d'été et d'hiver maintenues à $20^{\circ}$, $30^{\circ}$ et $37^{\circ} \mathrm{C}$. Pour ces deux catégories d'abeilles, la durée de vie moyenne est la plus longue à $30^{\circ}$ et la plus courte à $20^{\circ}$.

FREE et SPENCER-BOoTh (I960-I962) ont examiné l'effet des températures extrêmes. Ils signalent que les ouvrières supportent mieux le froid intense que les mâles et les reines; cependant, les ouvrières meurent après une immobilisation de $5^{\circ} \mathrm{h}$ à une température comprise entre $-2^{\circ}$ et $-6^{\circ} \mathrm{C}$. Les abeilles d'hiver, d'âge indéterminé, résistent mieux à cette immobilisation par le froid que les abeilles d'été. Elles supportent plus facilement les températures élevées $\left(40-50^{\circ} \mathrm{C}\right)$ à condition qu'elles aient été préalablement acclimatées à des températures telles que $35^{\circ} \mathrm{C}$ par exemple. Cependant, les jeunes abeilles non acclimatées présentent une mortalité réduite.

\section{e) État de la nourriture.}

Maurizio (I946) signale que la durée de vie moyenne d'abeilles d'été nourries de candi est de 25,2 jours. Elle est de 23,6 jours pour les mêmes abeilles nourries d'eau sucrée. Avec des abeilles d'hiver, la durée de vie moyenne est de 37,4 jours avec de l'eau sucrée et de 35,5 jours avec du candi. Elle pense que 1'aspect liquide ou solide de la nourriture a peu d'influence à condition que les abeilles reçoivent par ailleurs de l'eau pure.

\section{f) Rapports entre longévité, nutrition et état physiologique des abeilles.}

Bien que KELIER-KITZINGER (I935) ne soit pas parvenue à augmenter la durée de vie d'abeilles encagées en leur donnant comme nourriture du pollen mélangé à de l'eau sucrée, il s'est avéré depuis que le pollen administré seul ou en mélange dans du candi ou du miel prolonge la vie des ouvrières orphelines. Ce rapport entre l'effet du pollen et la longévité a été mis en évidence par plusieurs auteurs tels que 
Beutler et Offinger (I950), Giordani (I957), De Groot (i953), Melampy et MCGregor (I939), Maurizio (I946-I954), WAHL, (I956, Ig63).

Tous les pollens n'ont pas la même valeur alimentaire. Parmi les pollens les plus efficaces, MaUrizio (I954) cite ceux de Calluna vulgaris, Castanea sativa, Papaver rhoeas, Pirus sp., Salix sp., Trifolium pratense et repens. La façon de récolter les pollens est très importante : les pollens récoltés dans les trappes sous forme de pelotes sont plus actifs que ceux ramassés à la main. Le même auteur démontre, après interprétation statistique des résultats, qu'il existe un rapport direct entre la longévité et l'état physiologique des abeilles : lorsque le pollen accroît la longévité d'abeilles jeunes, il permet aussi le développement de leurs glandes hypopharyngiennes, des ovaires et des corps adipeux. La corrélation est surtout marquée entre la longévité et 1'état du corps adipeux organe de stockage des protéines, des lipides et du glycogène. Lorsque les abeilles sont âgées de plus de I5 jours, la longévité et l'état physiologique ne sont plus influencés par la nature de l'alimentation.

L'action du pollen sur l'état physiologique avait déjà été remarquée par d'autres auteurs : BACK (I956), GIORDANI (I957), HEJTMANEK (I933-I943), LOTMAR (I939), Mussbichler (I952), Pain (I961), Peterka (I939), Svoboda (I940), Svoboda et Péterka (I937), WAHL (I963).

La plupart des auteurs déjà cités, exception faite de Soudek (I927), Meinampy, MCGREGOR (I939), BEuTrER et OPFINGER (I950) s'accordent pour signaler que le pollen peut être remplacé par un certain nombre de succédanés dont certaines caséines et farines de soja, le lait écrémé en poudre et certaines levures. Ils peuvent, dans une certaine mesure, remplacer le pollen et augmenter la durée de vie des ouvrières.

Les résultats acquis s'accordent pour démontrer le rôle important de l'azote dans les pollens et les succédanés.

I,es pollens considérés comme très actifs sur 1'augmentation de la durée de vie ont une teneur en azote plus élevée que les pollens peu actifs, tels que les pollens anémophiles (Maurizio, I954). Les abeilles choisissent d'ailleurs dans une certaine mesure les pollens en fonction de leur richesse en azote (LouvEAUx, I958). Si des jeunes abeilles sont privées d'azote, elles vivent aux dépens de leurs propres réserves protéiques et la teneur en azote de la tête et de l'abdomen baisse plus que celle du thorax. La teneur du corps en azote redevient normale après apport d'azote alimentaire (HAYDAK, I937).

En ce qui concerne l'action des acides aminés, DE Groot (I953) a montré qu'avec des mélanges de ceux-ci, les longévités sont plus faibles que celles obtenues avec des protéines intactes ou hydrolysées.

Le pollen contenant des vitamines, dont celles du groupe B, leur action a été aussi examinée du point de vue de la longévité. Selon DE GROO'̇ (I95I), MaurrzIO (I954) elles n'auraient pas d'effet sur la durée de vie. Cependant, BAcK (I956) indique que de petites quantités de vitamines ajoutées à la caséine ont une légère action positive. Les abeilles élèvent plus facilement du couvain et les abeilles écloses ont une durée de vie améliorée si les nourrices ont reçu des vitamines.

Nous ne parlerons pas ici des modifications biochimiques qui apparaissent avec 1'âge, modifications signalées par RocKsTEIN et d'autres auteurs.

En résumé, on peut dire qu'on dispose de données assez complètes sur la durée de vie des ouvrières. Cependant, quelques-unes restent encore contradictoires comme 
l'influence de l'activité des butineuses ou l'action de certains constituants de l'alimentation tels que les vitamines par exemple.

L’influence de la reine n'a été observée que par la présence du couvain qu'elle produit. Ce facteur étant reconnu comme l'un des plus importants lors des recherches sur des colonies en liberté.

Ein présence de petits groupes d'abeilles claustrées, les répercussions de l'influence d'une reine sur la vie des ouvrières sont restées jusqu'à présent dans l'ombre.

Il importait, selon nous, d'étudier ce nouveau problème, en tenant compte de la multiplicité des facteurs qui entrent en jeu, et en particulier du facteur alimentaire.

\section{MATÉRIEL, ETT MÉTHODE DE TRAVAII,}

\section{A - Le matíriel biologique}

I. les oworives.

Dans la majorité des as, nous avons employé des abeilles jeunes d'âge connu, nées en étuve a) la température de $32^{\circ}{ }^{\circ}$.

Pour obtenir ces abeilles, des cadres de couvain naissant sont prélevés soit l'été dans des colonies normales non chauffées, soit l'hiver clans des colonies maintenues à une température ambiante de $20^{\circ} \mathrm{C}$. I ans ce demier cas, les colonies reçoivent chaque jour $100 \mathrm{~cm}^{3}$ de sirop tiède à $50 \mathrm{p}$. 100 et une fois par mois $300 \mathrm{~g}$ de pollen conservé non séché il -24" C, distribué humecté de miel dans les cellules d'un ravon.

Les cadres de couvain sont prélevés lorsque les nymphes de couleur foncée sont déja fortement chitinisées sous les opercules.

l.es éclosions obtenues de chaque cadre sont contrôlées tous les jours. Matis, pour obtenir un nombre sulfisant d'abeilles dans les expériences nécessitant un grand nombre de cagettes contenant chacune plus de 50 individlus, nous avons utilisé parfois des ouvrières nées depuis 48 ou $7^{2}$ heures.

Nous ne nous sommes jamais servi d'abeilles naissant sur un cadre de couvain ayant séjourné plus de 6 jours à l'étuve. lin effet, nous nous sommes apercu que les abeilles provenant de cadres en fin d'éclosion sont somvent de plus petite taille que celles qui naissent les premières. Eilles ont, rle plus, des ailes souvent malformées.

Pour les expériences poursuivies en hiver, nous avons utilisé plusieurs cadres de couvain ne provenant pas obligatoirement d'une même ruche. I ans ce cas précis, avant la mise en cage, nous avons réuni toutes les abeilles en les secouant daus une des boîtes d'éclosion et en les laissant cnsuite se regrouper librement sur un ravon vide.

De cette façon, nous avions un nombre plus grand d'individus ainsi qu'une répartition plus homogène des âges et des états physiologiques.

Dans un cas, nous avons utilisé des abeilles d'âge indéterminé en provenance de colonies chauflées. Elles étaient choisies sur les cadres situés à côté du nid à couvain, de façon à éviter de prendre une proportion trop élevée d'abcilles jeunes.

I.ors d'essais préliminaires, nous avons réuni les reines avec soit I, soit 2, 5, 10, 20 on 30 ouvrières. Nous pensions ainsi mettre en évidence avec plus de facilité, l'action possible de la reine sur ces groupes dont la durée de vie est plus faible que celle de groupes supérieurs à zo ouvrières. Mais, méme en présence d'une reine, les ouvrières moururent très vite. 1)'autre part, les reines, en présence de moins de 30 abeilles. supportèrent très mal la captivité. Fresnaye (1965) vient d'ailleurs de montrer que la durée moyenne de vie de jeunes reines claustrées dans des caget tes expérimentales est de 35,2 jours dans les meilleures conditions lorsqu'elles sont accompagnées de 40 à 50 abeilles.

Nous avons donc choisi des effectifs supérieurs à 30 ouvrières, tels que 50,55 et 100 ouvrières, pour nous placer dans des conditions optima de survie pour les ouvrières et les reines.

\section{Li's reinles.}

Nous avons expérimenté avec des reines vivantes ou mortes ou avec leur extrait naturel ou avec la phérormone I de synthèse.

Pour nos premiers essais, nous nous sommes servi de jeunes reines vierges, toutes nées à l'étuve.

Cès reines, aussitôt après leur naissince, étaient mises en expérience accompagnées d'ouvrières : 
d'autres, vieillies en étuve, étaient gardées en présence de 40 à 50 accompagnatrices, ces dernières étant renouvelées chaque mois en attendant l'utilisation des reines.

Par la suite, nous avons choisi des reines fécondes. Des groupes d'ouvrières ont reçu des reines dites " hétérogènes " quant à leur âge et à leur race. Elles nous avaient été expédiées par les apiculteurs.

D'autres groupes ont reçu des reines fécondes homogènes telles que de jeunes reines de race italienne, prêtes à pondre, mises en expérience dès leur réception ou vieillies à l'étuve de la même manière que les reines vierges. Dans ces cas, l'acceptation des reines et leur introduction est facilitée en badigeonnant leur corps avec une solution diluée de miel.

Des reines mortes fécondes et attractives ont également fait l'objet de ces recherches. Avant de les mettre au contact des ouvrières, nous les avons sélectionnées quant à leur pouvoir d'attraction, suivant la méthode de PAIN (I96I) : ces reines furent jugées suffisamment attractives pour nos expériences lorsqu'un minimum de 1o jeunes ouvrières s'intéressaient à leur cadavre.

Nous signalerons ici la difficulté que nous avons cue à rassembler en même temps, d'une part des groupes de reines de même âge, nées dans les mêmes conditions et présentant des facteurs d'homogénéité suffisants quant à la race, l'attraction, l'origine et l'activité et, d'autre part, des groupes importants d'ouvrières jeunes.

L'action de la phérormone royale attractive contenue dans les glandes mandibulaires des reines a été envisagée en utilisant non pas les glandes, mais un broyat de têtes de jeunes reines fécondes, en solution dans l'éther. L'extrait éthéré de tête déposé sur une languette de papier-filtre est présenté aux ouvrières, après évaporation du solvant, tous les jours sur une période de to jours.

De la même façon, nous avons examiné l'action de la phérormone royale de synthèse ou phérormone I, en diluant dans l'éther l'acide céto-9 décène-2-trans oïque.

\section{$\mathrm{B}$ - Le matériel technique}

Les ouvrières orphelines témoins, aussi bien que celles possédant une reine, étaient introduites dans des cagettes expérimentales en Altuglas, de i i $\mathrm{cm}$ de haut, sur 9,5 de large et 7,5 de profondeur. Leur description détaillée est exposée par l'un de nous à la suite de ce travail. Ces cagettes étaient maintenues dans des étuves réglées à $33^{\circ} \mathrm{C}$. Les étuves étaient elles-mêmes placées en chambre climatisée à une température constante de $20^{\circ} \mathrm{C}$ et à $70 \mathrm{p}$. Ioo d’humidité relative.

\section{$\mathrm{C}-$ Méthode}

La répartition des abeilles dans les cagettes a été effectuée au hasard de la façon suivante : les cagettes sont disposées d'une manière quelconque auprès du cadre d'éclosion. A l'aide d'une pince souple, les insectes sur le rayon, sont saisis par le thorax et répartis un par un dans des cagettes différentes. Après avoir réuni Io abeilles par cage, nous avons fait varier la disposition des cagettes et l'ordre d'introduction des abeilles. Cette méthode est assez longue et demande de la part de I'opérateur une grande attention. Elle a pour avantage de réaliser une homogénéité quant à l'âge des ouvrières prélevées sur le cadre.

Dans le cas d'ouvrières d'âge indéterminé, nous avons opéré devant une fenêtre, dans une chambre à $18^{\circ}$. Les abeilles, attirées par la lumière du jour, ont été capturées sur la vitre et introduites de la même façon dans les cagettes expérimentales.

Chaque expérience comprend deux lots de cagettes : un lot témoin comprenant plusieurs cagettes d'ouvrières orphelines et un lot traité comprenant le même nombre de cagettes peuplées d'ouvrières avec reine.

Pour toutes ces expériences, la nourriture de base fut le candi, composé de miel et de sucre glace dans la proportion $1: 3$. De l'eau était donnée ad libitum. Dans les cas où nous avons offert du pollen, celui-ci provenait d'un même stock constitué par un mélange de récoltes non séchées conservées à la température de $-24^{\circ} \mathrm{C}$. Le pollen était administré seul, à part du candi, dans de petites cupules en matière plastique à bords hauts, selon la technique de PAIN (I96I). Cependant, au cours de ces expériences, les abeilles qui reçurent du pollen ne furent jamais affamées par suppression du candi.

Chaque jour, la propreté de la nourriture était contrôlée; le nettoyage des cagettes était effectué; chaque pièce mobile salie était changée. De même, les abeilles mortes étaient retirées des cagettes tous les jours et dénombrées cage par cage et lot par lot. Nous signalerons au passage que nous n'avons pu mener ces différentes expériences au cours d'une même année apicole.

Nous n'avons pas jugé utile de poursuivre les expériences jusqu'à l'extinction totale des lots d'abeilles parce qu'il nous apparaît que, passé un certain temps d'encagement de l'ordre de 30 jours, les ouvrières se trouvent dans de mauvaises conditions de confinement et présentent un état physiologique aberrant qui perturbe les observations. Des expériences ont donc été terminées au bout de ce laps de temps, alors que d'autres se sont prolongées jusqu'à $34^{-25}$ jours et même jusqu'à une 
quarantaine de jours. Les expériences dans lesquelles les ouvrières recevaient du pollen ont duré nettement plus longtemps (exp. $n^{\text {os }}$ I, 3, 5). Cette méthode qui peut ne pas paraître logique a pourtant sa raison d'être. En effet, chaque fois que nous avons décidé d'arrêter une expérience, c'est parce que nous avons remarqué une modification dans le comportement des insectes portant surtout sur une augmentation importante de la production des déjections.

\section{RÉSULTATS}

Nous exposerons maintenant les différentes expériences et nous interprèterons les courbes obtenues pour chacune d'elles. Celles-ci ont été tracées en notant chaque jour les mortalités des 2 lots puis en les cumulant dès le $2^{\mathrm{e}}$ jour et jusqu'à la fin des expériences. Le tableau I résume l'ensemble de ces données.

\section{Expérience $n^{0} 1$.}

L'expérience $\mathrm{n}^{\circ} \mathrm{I}$, effectuée en été, porte sur 2 lots de 6 cages; chacune d'elles contient 55 ouvrières d'un jour, soit 350 abeilles par lot.

Une reine vierge de quelques jours est introduite dans chacune des cages du lot traité. Au cours des 46 jours d'expérience, toutes les cagettes sont pourvues en pollen.

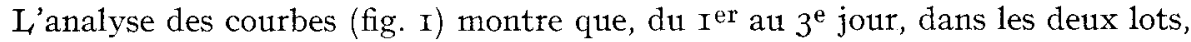
les mortalités sont très faibles et comparables. A partir du $4^{\mathrm{e}}$ jour et jusqu'au $32^{\mathrm{e}}$, les mortalités chez les ouvrières orphelines sont supérieures à celles des ouvrières avec reine.

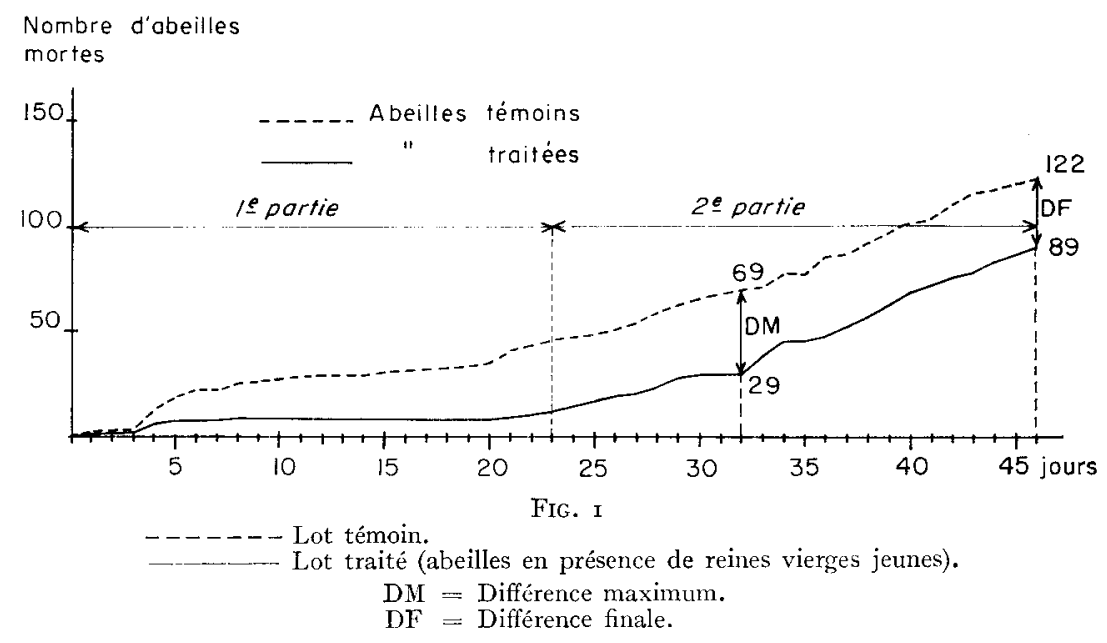

La différence maximum de mortalité entre les 2 lots est, au $32^{\mathrm{e}}$ jour, de 40 abeilles soit I2 p. Ioo de plus d'abeilles mortes chez les témoins.

A partir $\mathrm{du} 3^{2} \mathrm{e}$ jour, cette différence diminue progressivement. Les courbes, bien que toujours parallèles, se rapprochent insensiblement.

A la fin de 1'expérience, au $46^{\mathbf{e}}$ jour, nous avons encore une différence de mortalité de 33 abeilles, soit ro p. Ioo de plus chez les témoins.

Étant donné que la courbe des témoins est située au-dessus de celle des traités, nous considérons qu'il existe une tendance positive de la reine à réduire les mortalités 
chez les ouvrières qui se trouvent à son contact. I a présence de pollen dans la nourriture a certainement réduit les mortalités dans les 2 lots ainsi que l'écart des courbes de mortalité.

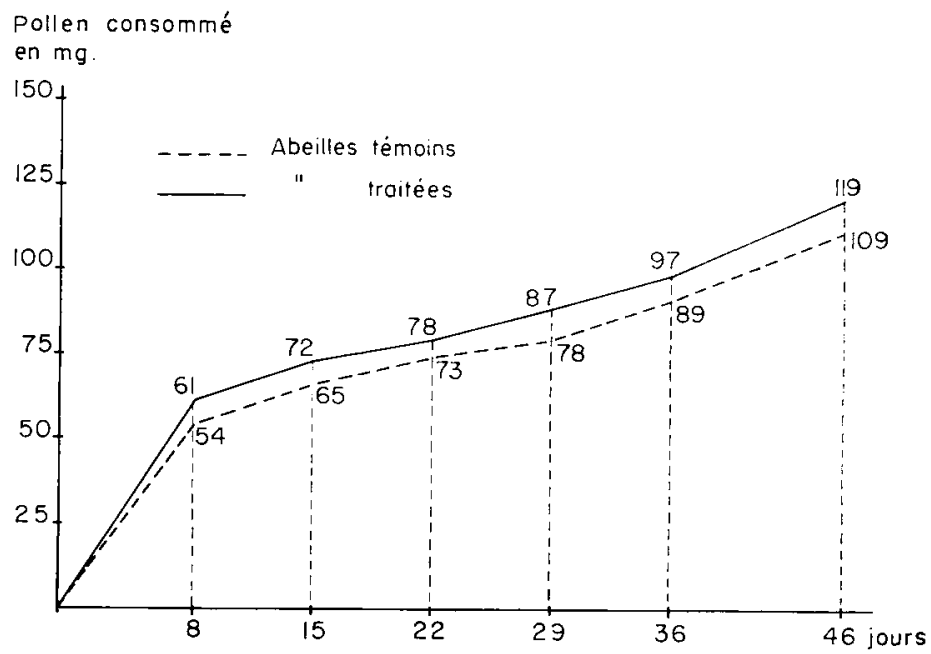

Fis, I bis.

Consommation de pollen pour une abeille témoin. Consommation de pollen pour une abeille traitée.

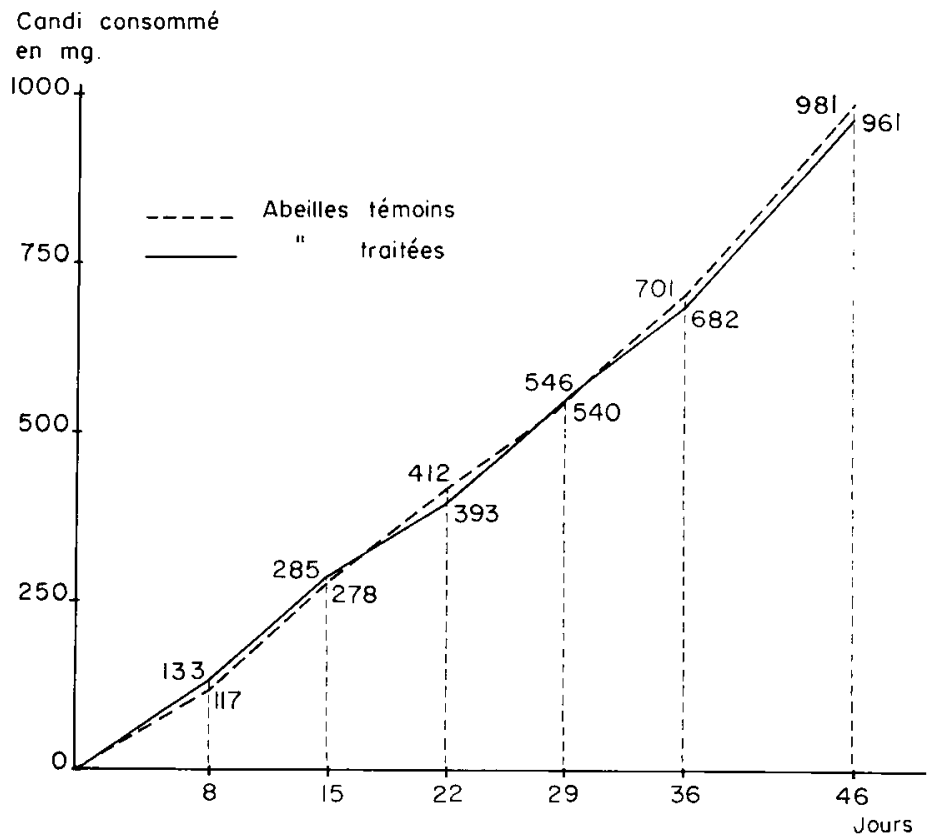

FIG. I ter.

Consommation de candi pour une abeille témoin.

Consommation de candi pour une abeille traitée. 
Pour cette expérience seulement, la consommation des aliments sucrés (candi) et celle de l'aliment azoté (pollen) ont été déterminées pour les deux lots.

L,es courbes (fig. I bis et I ter) représentent les consommations cumulatives mesurées tous les 7 jours sauf au début ( 8 jours) et en fin d'expérience (Io jours). Elles ont été rapportées à $I$ abeille par lot en tenant compte des mortalités.

Elles montrent que chez une abeille traitée, la consommation en pollen augmente progressivement et reste toujours supérieure à celle des ouvrières témoins.

Par contre, la consommation en candi est la même, bien que légèrement supérieure en fin d'expérience chez l'abeille témoin.

L'influence de la reine semble surtout avoir porté sur la prise de nourriture azotée. Des expériences sont à poursuivre dans cette direction.

\section{Expérience no 2.}

L'expérience $\mathrm{n}^{0} 2$ se rapproche de la précédente mais, si le nombre de cages par lot reste le même, le nombre d'ouvrières par cage est de Ioo, soit 600 par lot

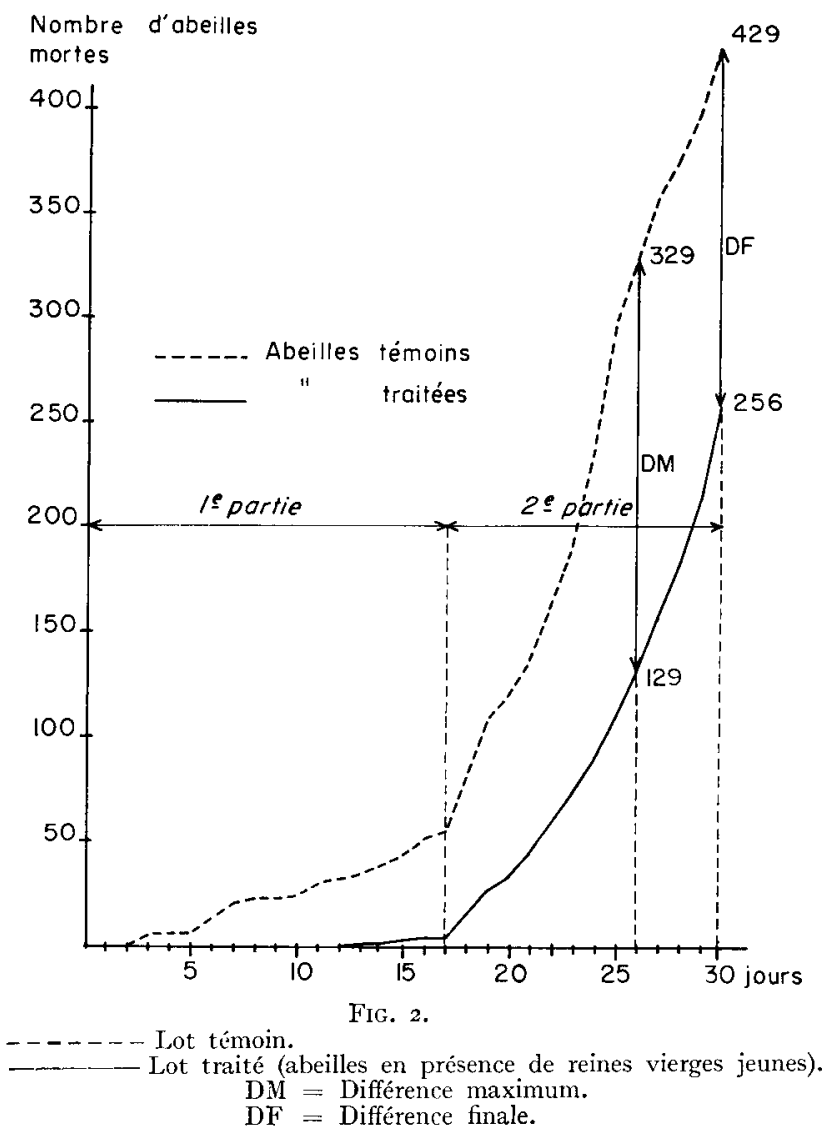

Il s'agit d'abeilles âgées de I à 3 jours. Les reines utilisées dans cette expérience sont comparables à celles de l'expérience I. Au cours des 30 jours de l'expérience, les cagettes n'étaient pourvues cette fois que de candi. L'analyse des courbes (fig. 2) 
montre que, dans le lot témoin, la mortalité débute le $3^{\mathrm{c}}$ jour, alors que dans le lot traité, elle ne débute qu'au $3^{3}$ jour. La différence de mortalité entre les 2 lots est, à ce moment-là, de 30 abeilles, soit 5 p. Ioo de plus d'abeilles mortes chez les témoins sans reine. Cette différence de mortalité ne cesse de croître, pour atteindre son maximum au 26 e jour où l'écart est de 200 abeilles, soit $33 \mathrm{p}$. roo de plus chez les témoins. A partir du $27^{\mathrm{e}}$ jour, l'écart diminue.

A la fin de l'expérience, au $30^{\mathrm{e}}$ jour, nous avons encore une différence de mortalité de I73 abeilles, soit 28 p. Ioo de plus chez les ouvrières orphelines.

Comme dans le premier cas, la courbe de mortalité des témoins se trouve située au-dessus de celle des traitées. Ici, les mortalités sont plus importantes car à partir du $I 7^{\mathrm{e}}$ jour, les 2 courbes s'élèvent rapidement alors que dans l'expérience $\mathrm{I}$, elles s'écartent très peu de l'abscisse, indiquant une mortalité très réduite, même en fin d'expérience.

La suppression du pollen, bien qu'elle augmente les mortalités, montre de façon plus nette l'influence de la reine qui se manifeste après le $\mathrm{I} 7^{\mathrm{e}}$ jour d'encagement. La différence maximum de mortalité entre les 2 lots est de I 2 p. Ioo dans l'expérience $\mathrm{I}$ et de $33 \mathrm{p}$. Ioo dans 1'expérience 2 .

Expérience $n^{\circ} 3$.

L'expérience $\mathrm{n}^{\circ} 3$ comprend 2 lots de 9 cages ; chacune d'elles renferme 55 ouvrières de 3 à 4 jours prélevées en automne dans des ruches chauffées. I 3 reines vierges, nées le même jour, ont été vieillies en étuve et les 9 survivantes mises au contact des ouvrières après 82 jours de captivité.

Comme nous voulions que l'expérience porte sur un très grand nombre de jours, nous avons administré du pollen à toutes les ouvrières. L'expérience s'est échelonnée sur 73 jours. Dès le $3^{\mathrm{e}}$ jour, la mortalité dans le lot témoin augmente par rapport au lot des abeilles traitées. Au $5^{\mathbf{e}}$ jour, une différence de I $5_{5}$ abeilles est enregistrée, soit 3 p. Ioo de plus d'abeilles mortes chez les ouvrières orphelines. Les courbes (fig. 3 ) demeurent pratiquement parallèles jusqu'au $27^{\mathrm{e}}$ jour où la différence est de 20 abeilles. A partir du $28^{\mathrm{e}}$ jour, les 2 courbes s'écartent de plus en plus. Au $44^{\mathrm{e}}$ jour, leur écart est à son maximum avec I23 ouvrières, soit $25 \mathrm{p}$. Ioo de plus d'abeilles mortes chez les témoins.

Puis, les courbes se rejoignent progressivement, bien qu'au $73^{\mathrm{e}}$ jour on note encore une différence de 25 abeilles, soit $5 \mathrm{p}$. roo de plus chez les témoins. La courbe de mortalité des témoins est toujours située au-dessus de celle des traitées. Malgré la présence de pollen, l'influence de la reine se manifeste plus clairement que dans l'expérience $\mathbf{I}$.

I'un de nous a déjà signalé (PAIN, I959) que plus les reines sont âgées, plus elles exercent un pouvoir d'attraction sur les ouvrières. Ici, les reines vierges âgées ont eu davantage d'influence sur la diminution de la mortalité des ouvrières accompagnatrices que les reines vierges jeunes.

Expérience $n^{\circ} 4$.

L'expérience $n^{\circ} 4$ comprend 2 lots de I9 cagettes contenant chacune 50 ouvrières d'été de I à 3 jours, alimentées uniquement de candi, pendant une période de 30 jours. Disposant d'un nombre relativement important de reines fécondes de rebut d'âge et d'origine inconnus, nous les avons introduites sans les endormir auprès 
TAUX DE MORTAITTÉ DES OUVRIÈRES

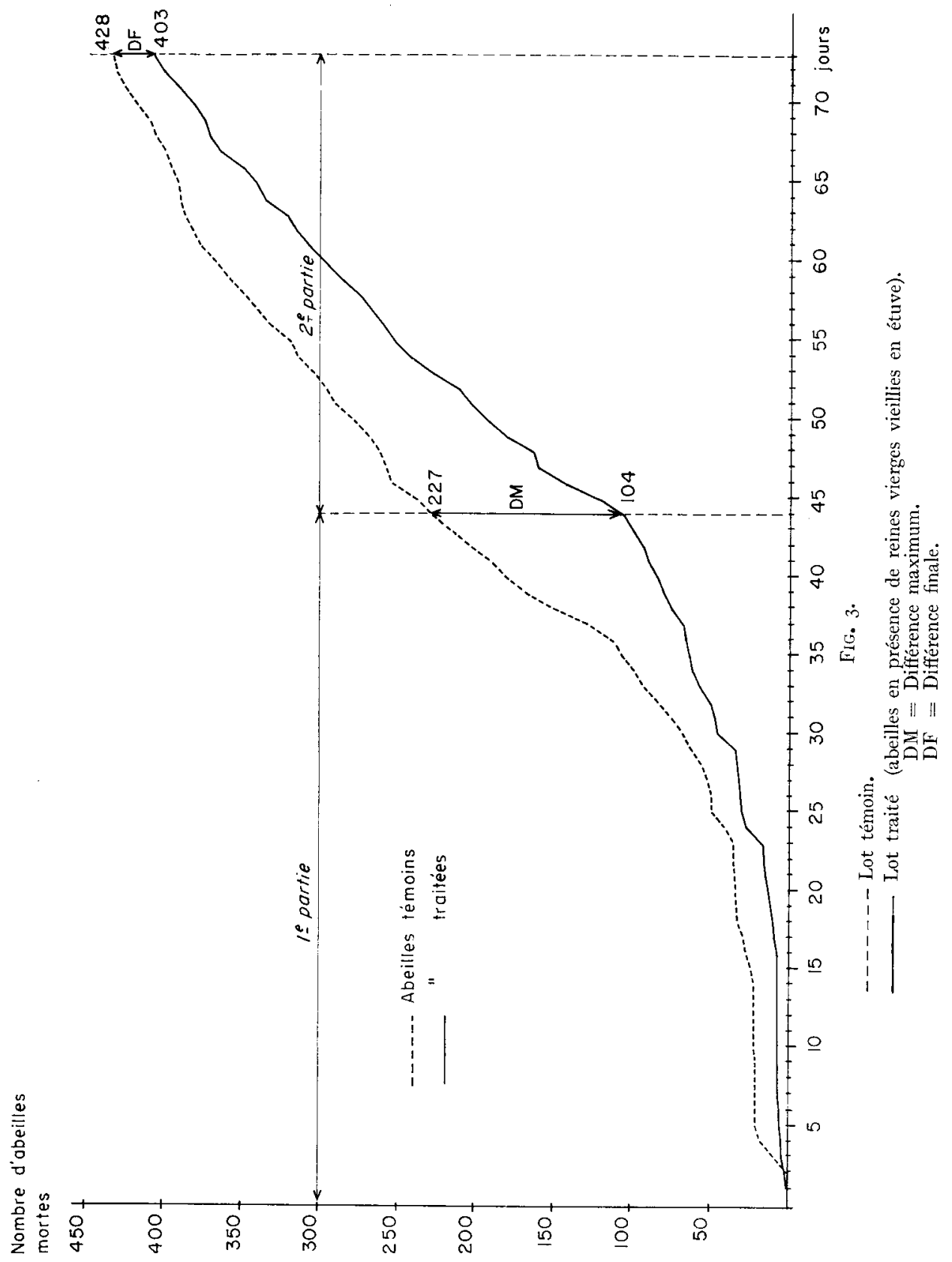


des ouvrières du lot traité. $\mathrm{Au} 4^{\mathrm{e}}$ jour, la mortalité des abeilles sans reine est plus forte que dans le lot traité. Jusqu'au Ig jour, les mortalités augmentent dans les deux lots mais les courbes restent parallèles et rapprochées l'une de l'autre (fig. 4).

A partir du $20^{\mathrm{e}}$ jour, les courbes s'écartent légèrement, puis se rapprochent et s'écartent à nouveau, pour atteindre une différence maximum de $8 \mathrm{I}$ abeilles au $29^{\mathrm{e}}$ jour, soit $8,5 \mathrm{p}$. roo de plus d'abeilles mortes chez les témoins. Au $30^{\mathrm{e}}$ jour, la différence est de 65 abeilles, soit 7 p. Ioo de plus chez les témoins. Sans pollen, les courbes s'élèvent rapidement comme dans l'expérience 2 . L'écart maximum des expériences 2 et 4 est situé à peu près à la même période, mais il est très faible ici.

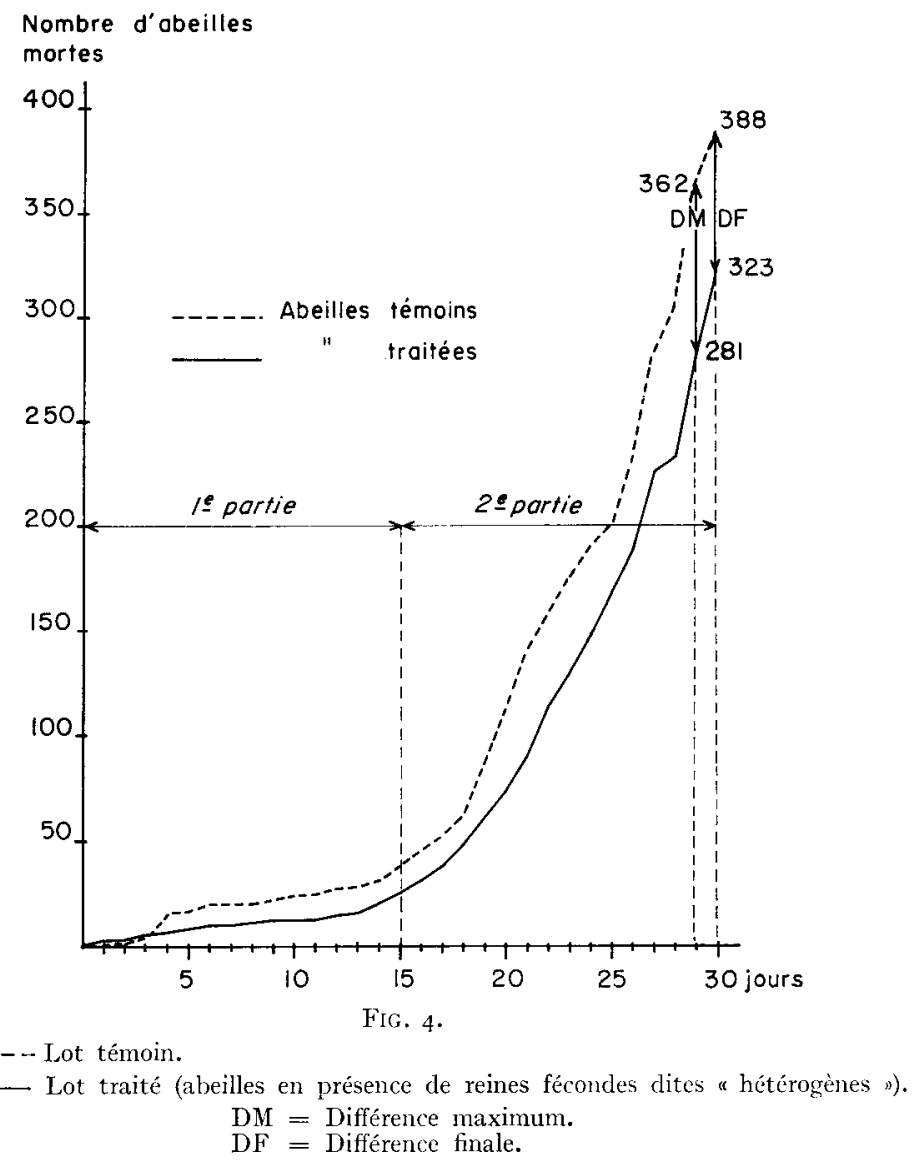

Cependant, la courbe de mortalité des traitées est toujours au-dessous de la courbe des témoins. Ainsi, même des reines de rebut influent légèrement sur le taux de mortalité des ouvrières. La différence peu accusée du taux de mortalité des deux lots traduit bien 1'hétérogénéité des reines utilisées.

Expérience no 5.

L'expérience $\mathbf{n}^{0} 5$ porte sur 2 lots de Io cagettes renfermant chacune 55 abeilles de 2 à 3 jours. Le couvain provient de ruches chauffées en hivernage. Pour prolonger 


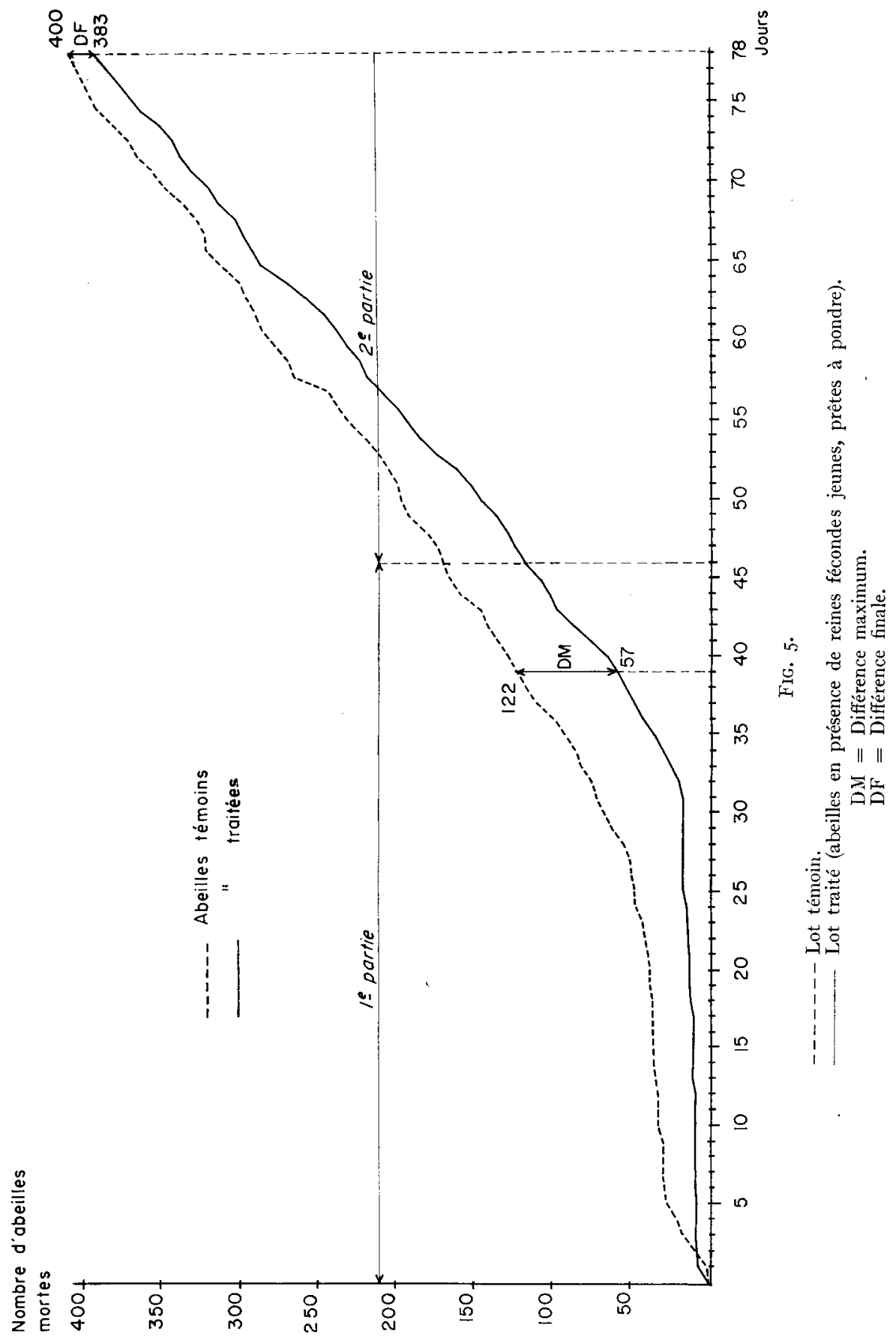


les observations, nous avons donné en nourrissement aux ouvrières encagées du pollen. De ce fait, 1'expérience s'est poursuivie 78 jours. Les reines employées sont toutes de race Italienne, jeunes et prêtes à pondre. Du $3^{\circ}$ au $3^{\mathrm{e}}$ jour, la mortalité est très réduite chez les abeilles traitées. Chez les abeilles témoins, la mortalité, bien que réduite, est cependant supérieure (fig. 5). Après le $30^{\mathrm{e}}$ jour, dans les deux lots, la mortalité est plus importante, mais elle est encore supérieure dans le cas du lot témoin. Au $39^{\mathrm{e}}$ jour, la différence maximum de mortalité est de 65 abeilles, soit I2 p. Ioo de plus d'abeilles mortes chez les témoins. Après le $40^{\circ}$ jour, les écarts de mortalité entre abeilles témoins et traitées se réđuisent et les courbes se rapprochent. Au $7^{8 \mathrm{e}}$ jour, la différence n'est plus que de I7 abeilles, soit 3 p. roo de plus chez les témoins. Les courbes se trouvent dans les mêmes situations, ce qui permet d'avancer que, du $30^{\mathrm{e}}$ jour à la fin de l'expérience, les reines exercent une influence positive sur la réduction du taux de mortalité des ouvrières. On peut dire que ces reines, en présence de pollen, ont exercé la même action sur les ouvrières que les jeunes reines vierges de $1^{\prime}$ expérience $\mathrm{n}^{\circ} \mathrm{I}$.

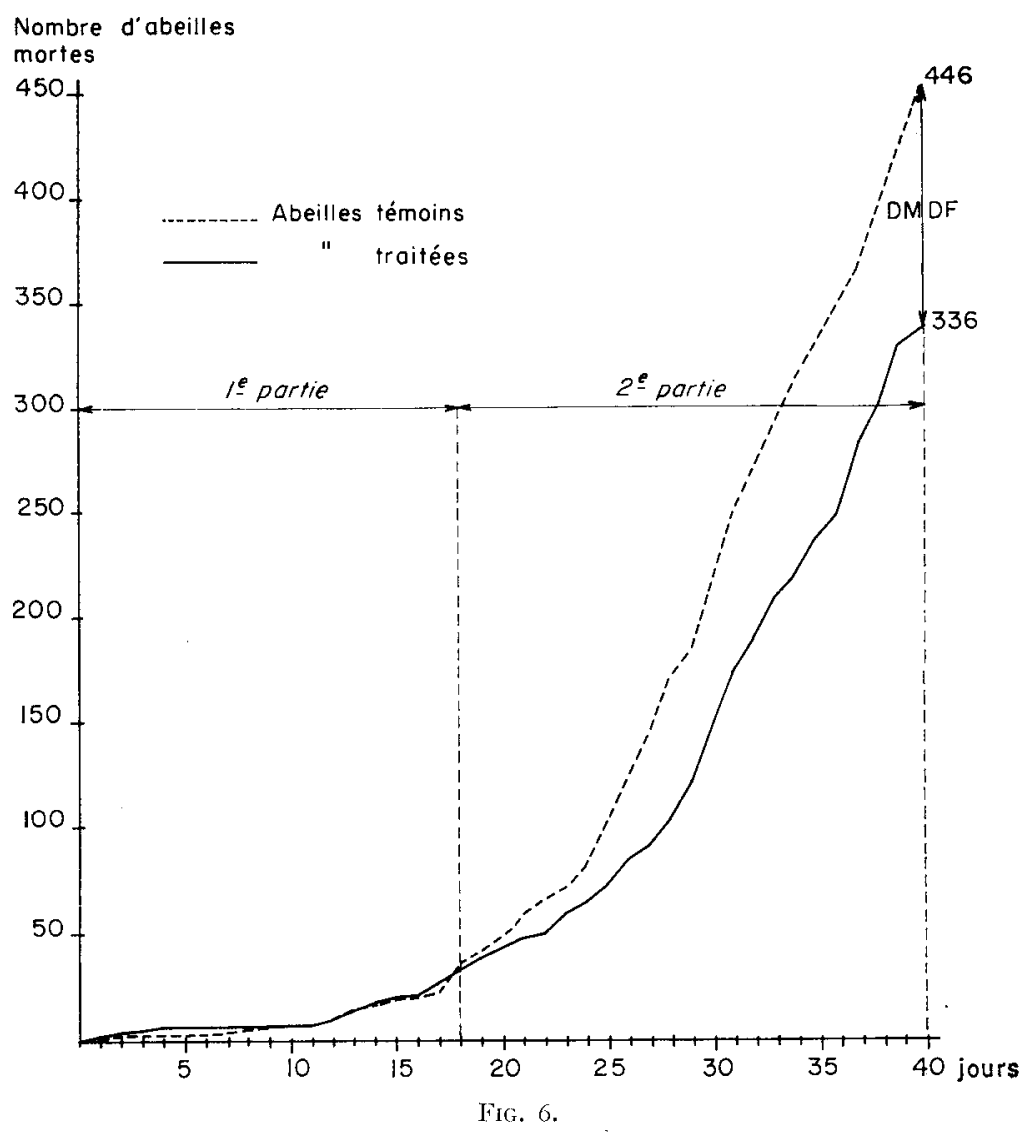

Lot témoin.

Lot traité (abeilles en présence de reines fécondes vieillies en étuve).

$\mathrm{DM}=$ Différence maximum.

$\mathrm{DF}=$ Différence finale. 
Expérience $n^{\mathrm{0}} 6$.

L'expérience $\mathrm{n}^{\circ} 6$ comporte 2 lots de 9 cagettes de roo abeilles chacune. Les ouvrières, d'âge indéterminé, proviennent de ruches chauffées, ne recevant que du candi.

Comme dans l'expérience $n^{\circ} 3$, les reines fécondes ont été vieillies en étuve et mises auprès des ouvrières après r 26 jours d'encagement. L'expérience a duré 40 jours. I'aspect des courbes (fig. 6) montre une mortalité comparable et peu importante des 2 lots, du I er au I 8 e jour. Les deux courbes se superposent. Puis la mortalité, chez les témoins, augmente progressivement jusqu'au dernier jour oì la différence est maximum avec I Io abeilles, soit I 2 p. Ioo de plus d'abeilles mortes chez les témoins. Les résultats sont comparables à ceux de l'expérience $\mathrm{n}^{\circ} 4$ ò̀ les reines fécondes, dites hétérogènes, étaient aussi d'âge avancé. En analysant les courbes de ces deux expériences $\left(\mathrm{n}^{0} 4\right.$ et $\left.\mathrm{n}^{0} 6\right)$, on s'aperçoit que, dans l'expérience $n^{\circ} 6$ les mortalités chez les témoins et chez les traitées sont plus faibles. La différence finale des mortalités est plus grande. Vu l'écart entre les 2 courbes, nous supposons que les reines fécondes utilisées ont exercé une influence légèrement plus grande que celle de l'expérience $n^{\circ} 4$, d'autant plus que les ouvrières accompagnatrices sont aussi plus âgées. Ces reines vieillies en étuve exercent cependant un effet beaucoup plus faible que les reines vierges âgées de l'expérience $\mathrm{n}^{0} 3$ qui reçurent du pollen.

\section{Expérience $n^{0} 7$.}

L'expérience $\mathrm{n}^{0} 7$ utilise I ooo abeilles par lot comprenant ro cagettes de Ioo abeilles chacune, soit au total 2 ooo ouvrières en provenance de ruches chauffées. Ce sont des abeilles de I à 2 jours, alimentées de candi pendant $4 \mathrm{I}$ jours.

F̂tant donné que dans toutes les expériences précédentes les reines vivantes utilisées ont réduit la mortalité des ouvrières groupées auprès d'elles, nous avons examiné quelle pouvait être l'influence de reines mortes et si celle-ci était de même importance que celle des reines vivantes. Les cadavres de reines furent choisis de même origine, parmi des reines fécondes. Du $\mathrm{I}^{\mathrm{er}}$ au $\mathrm{I}_{5}{ }^{\mathrm{e}}$ jour, chaque cagette du lot traité reçut un cadavre attractif de reine qui resta dans celle-ci pendant toute la durée de l'expérience. Puis, au $\mathrm{I}^{\mathrm{e}} \mathrm{e}$ jour, nous avons ajouté une $2^{\mathrm{C}}$ reine morte choisie selon les critères décrits plus haut, afin de pallier à l'épuisement de l'odeur des premières. Deux reines mortes ont donc séjourné du $\mathrm{I}^{\mathrm{e}}$ au $4 \mathrm{I}^{\mathrm{e}}$ jour. Les abeilles témoins reçurent une reine vierge, morte à la naissance et, par conséquent, non attractive. L'un de nous (PAIN, I959) a en effet démontré 1'absence de leur pouvoir d'attraction.

Les courbes (fig. 7) indiquent que, du $\mathrm{I}^{\mathrm{er}}$ au $\mathrm{I}^{\mathrm{e}}$ jour, la mortalité entre les deux lots est semblable. Au-delà du Ige jour, la mortalité du lot témoin sans reine augmente progressivement par rapport à celle du lot traité. En fin d'expérience, au $4 \mathrm{I}^{\mathrm{e}}$ jour, nous avons une différence maximum de mortalité entre les deux courbes de I 85 abeilles, soit I 8,5 p. Ioo de plus d'abeilles mortes chez les ouvrières orphelines.

La mortalité che $\%$ les témoins est toujours la plus forte; les courbes présentent la même allure, celle des traitées restant toujours au-dessous de celle des témoins. L'influence des reines mortes est plus importante que celle des reines fécondes vivantes vieillies en étuve. Mais ces dernières ont été mises au contact d'ouvrières 
d'âge indéterminé, ce qui a eu pour effet d'augmenter les mortalités chez les traitées. On peut en partie comparer l'action des cadavres de reines à celle des jeunes reines vierges vivantes de l'expérience $\mathrm{n}^{\circ} 2$ bien qu'elle s'exerce plus tardivement.

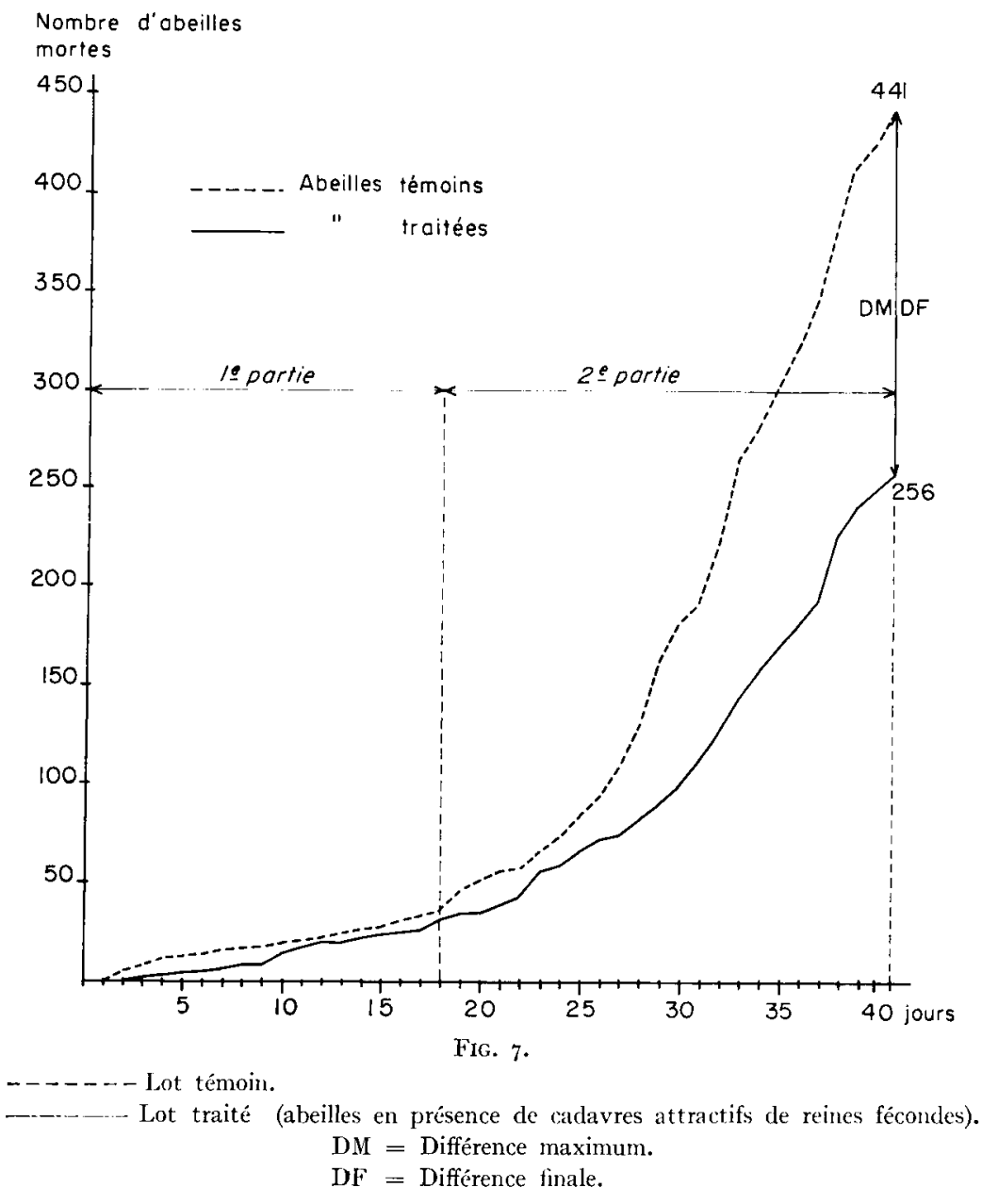

Expérience $n^{\circ} 8$.

L'expérience $n^{\circ} 8$ porte sur un lot traité de Io cagettes et un lot témoin de 9 cagettes, 1'une des cagettes témoins ayant été supprimée à cause de la très forte mortalité observée dès le $2^{\mathrm{e}}$ jour et d'origine purement accidentelle. Cent abeilles d'automne de I à 2 jours ont été réparties dans chacune des cagettes et ont reçu aussitôt du candi. L'expérience a duré 35 jours.

Les reines mortes s'étant révélées intéressantes, nous avons essayé de localiser le facteur influent. Nous avons pensé qu'il était peut-être en rapport avec le pouvoir d'attraction que manifestent la plupart des reines. Leur tête étant plus particulièrement attractive (PAIN, I954) que le reste du corps, nous avons préparé un broyat de 30 têtes de jeunes reines fécondes en solution dans $50 \mathrm{ml}$ d'éther. Il contenait 


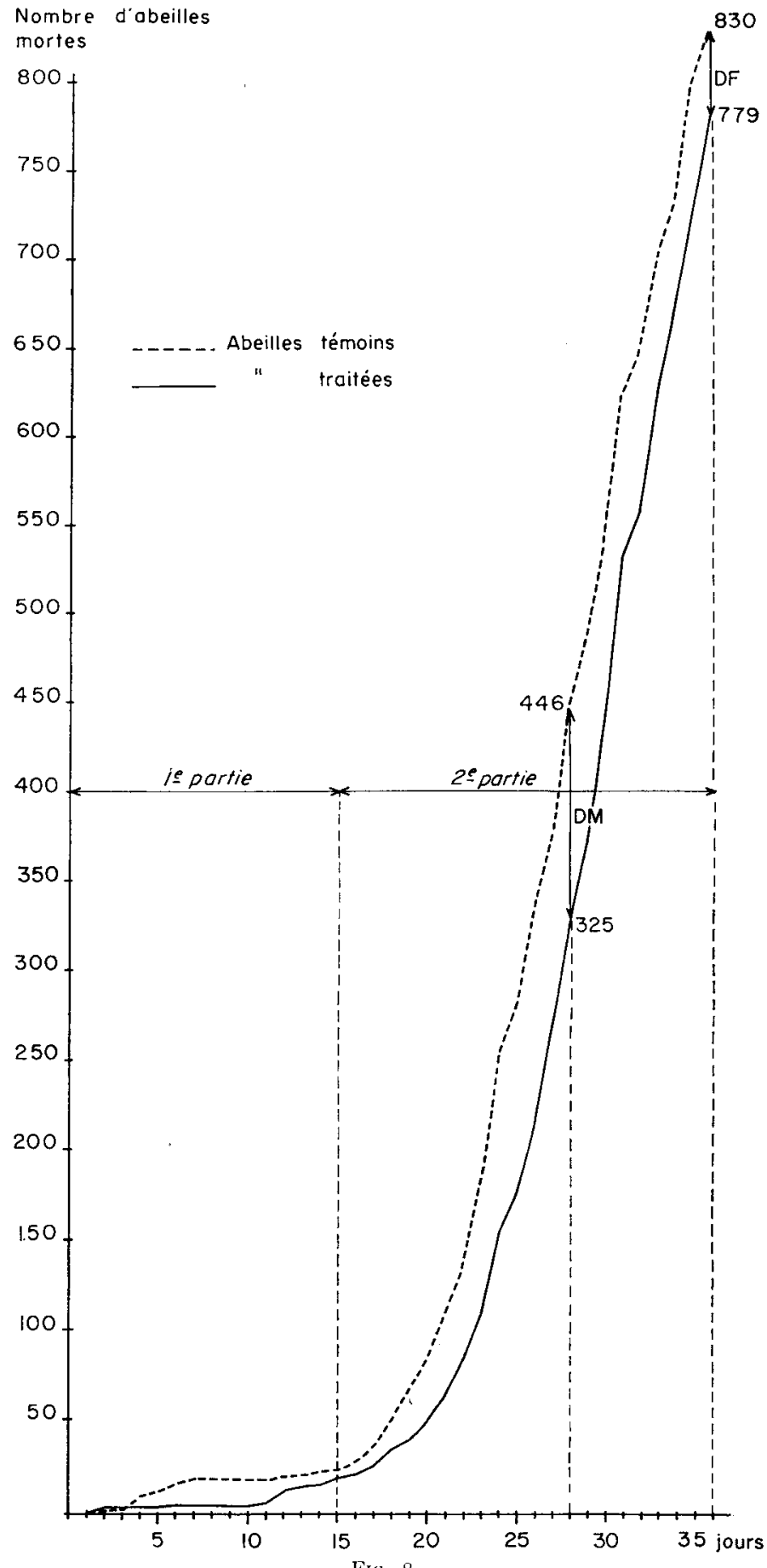

FIG. 8.

Lot témoin

Lot traité (abeilles en présence de broyat de têtes de ieunes reines fécondes). $\mathrm{DM}=$ Différence maximum.

$\mathrm{DF}=$ Différence finale. 
donc l'extrait des glandes mandibulaires responsable de l'attraction. Après vérification du degré de celle-ci, chaque cagette du lot traité reçut, pour une durée totale de Io jours, 1'équivalent de 3 têtes de reines déposé sur papier-filtre, soit 5/Ioo de ml, par cage et par jour. Les cagettes témoins reçurent un papier imbibé d'éther mais sans broyat céphalique. Lorsque les papiers étaient détériorés, des papiers neufs étaient introduits.

L'analyse des courbes (fig. 8) montre que les mortalités sont pratiquement identiques jusqu'au i $\delta^{\mathrm{e}}$ jour. A compter du $\mathrm{I} 9^{\mathrm{e}}$ jour jusqu'au $28^{\mathrm{e}}$, la mortalité est rapide dans les 2 lots. Les 2 courbes s'élèvent rapidement. Cependlant la mortalité est plus importante chez les témoins. La différence maximum se situe au $28 \mathrm{e}$ jour avec I 2 I ouvrières, soit I 2 p. Ioo de plus d'abeilles mortes chez les témoins. Après le $28 \mathrm{e}$ jour, les mortalités décroissent progressivement dans les 2 lots. Au dernier jour, la différence de mortalité n'est plus que de 5 I ouvrières soit $5 \mathrm{p}$. Ioo seulement de plus chez les témoins.

Les abeilles traitées meurent dans l'ensemble moins vite que les abeilles ne recevant pas de broyat de têtes de reines. La courbe témoin est encore au-dessus de celle des traitées.

\section{Expérience no 9.}

Dans l'expérience $n^{\circ} 9$, nous disposons de 12 cagettes contenant chacune 50 ouvrières de printemps de I à 3 jours, alimentées de candi. L'expérience s'étage sur 34 jours.

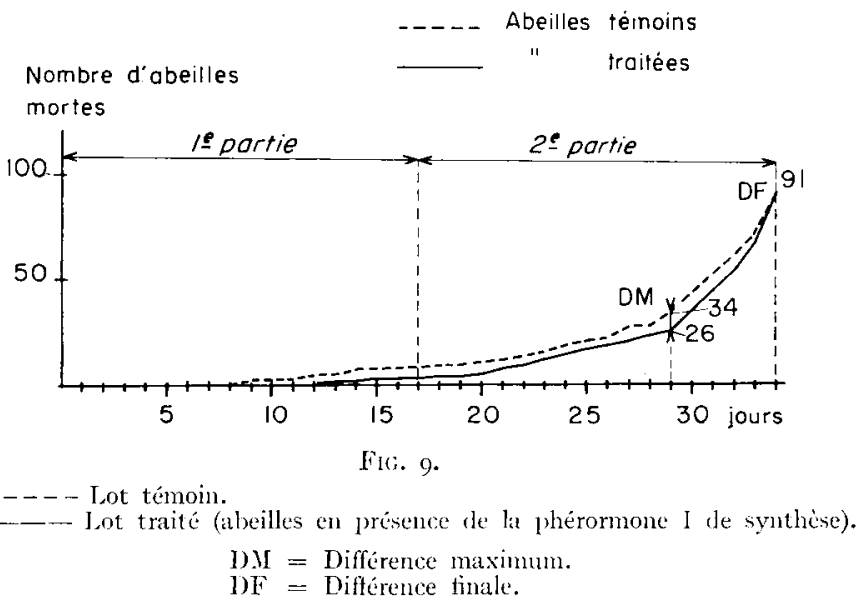

Bien que l'expérience précédente n'ait pas clonné de résultats aussi nets que ceux de l'expérience $n^{0} 7$, mais seulement une tendance positive de la part des broyats de tête de reine, nous avons utilisé l'une des substances contenues dans l'extrait royal mandibulaire, dont la synthèse en France a été mise au point par BARBIER et LEDERER ( 1960 ). Il s'agit de 1'acide céto-9 décène-2-trans oïque, encore appelé phérormone I. Chaque cagette du lot traité reçoit I fois par jour, sur une clurée de Io jours, en solution dans l'éther froid, i Io ug d'acide déposé sur une languette de papier. Après évaporation, nous ajoutons sur les papiers une mince pellicule de 
miel. Les papiers ainsi préparés sont offerts aux ouvrières préalablement affamées durant I heure. Les doses d'acide ont été calculées en sachant que des reines vierges, à leur mort, contiennent I30 $\mu \mathrm{g}$ de cet acide (BUTLER et PATON, Ig62). L'existence de variations quant à ces quantités (PAIN, non publié) nous a fait utiliser une dose plus forte, telle que I,I mg administrée au total dans une cagette.

Dans ce cas, les courbes (fig. 9) sont très rapprochées et toujours parallèles. La différence maximum n'est que de 2 p. Ioo au $29^{\mathrm{e}}$ jour. Au $34^{\mathrm{e}}$ jour, les mortalités sont identiques.

Malgrê l'écart minime entre les deux courbes, celle des ouvrières témoins ne recevant pas d'acide est encore placée au-dessous de celle des ouvrières traitées.

\section{Analyse statistique des résultats (1)}

Pour l'interprétation statistique des résultats, nous avons appliqué le test $\mathrm{F}$ de Snedecor. Il porte sur les chiffres bruts d'abeilles mortes notés au cours de la durée totale de l'expérience. Dès le premier jour de celle-ci, le nombre d'abeilles mortes est totalisé pour chacun des lots. Puis les mortalités des jours suivants sont cumulées successivement jusqu'à la fin de l'expérience.

On effectue alors la somme totale $(\Sigma x)$ des mortalités cumulées au jour le jour, pour un lot et pour un nombre de jours donnés. Soit $\Sigma x_{1}$ pour le lot témoin et $\Sigma x_{2}$ pour le lot traité (abeilles avec reine).

On établit d'une part la somme de $\Sigma x_{1}+\Sigma x_{2}$ et, d'autre part, la somme de $\Sigma x_{1}^{2}+\Sigma x_{2}^{2}$.

On introduit un terme de correction :

$$
\frac{\left(\Sigma x_{1}+\Sigma x_{2}\right)^{2}}{N},
$$

( $N$ étant égal au nombre de jours où l'on a enregistré les mortalités dans les 2 lots).

La variation totale est égale à la différence entre la somme totale des mortalités cumulées élevées au carré et le terme de correction :

$$
\Sigma x_{1}{ }^{2}+\Sigma x_{2}{ }^{2}-\frac{\left(\Sigma x_{1}+\Sigma x_{2}\right)^{2}}{N} .
$$

La variation entre les groupes est représentée par l'expression :

$$
\frac{\left(\sum x_{1}\right)^{2}}{n_{1}}+\frac{\left(\sum x_{2}\right)^{2}}{n_{2}}-\frac{\left(\sum x_{1}+\sum x_{2}\right)^{2}}{N},
$$

( $n$ étant égal au nombre de jours où l'on a enregistré les mortalités dans un lot).

La variation à l'intérieur des groupes s'exprime par la différence entre la variation totale et la variation entre les groupes. L,e nombre de degrés de liberté est égal à la somme du nombre des données de chaque groupe, diminué chaque fois de un, soit $\nu=n_{1}-\mathrm{I}+n_{2}-\mathrm{I}$.

(1) Nous remercions MM. Vergaron et Jerry qui ont participé à l'étude statistique de ces données. 


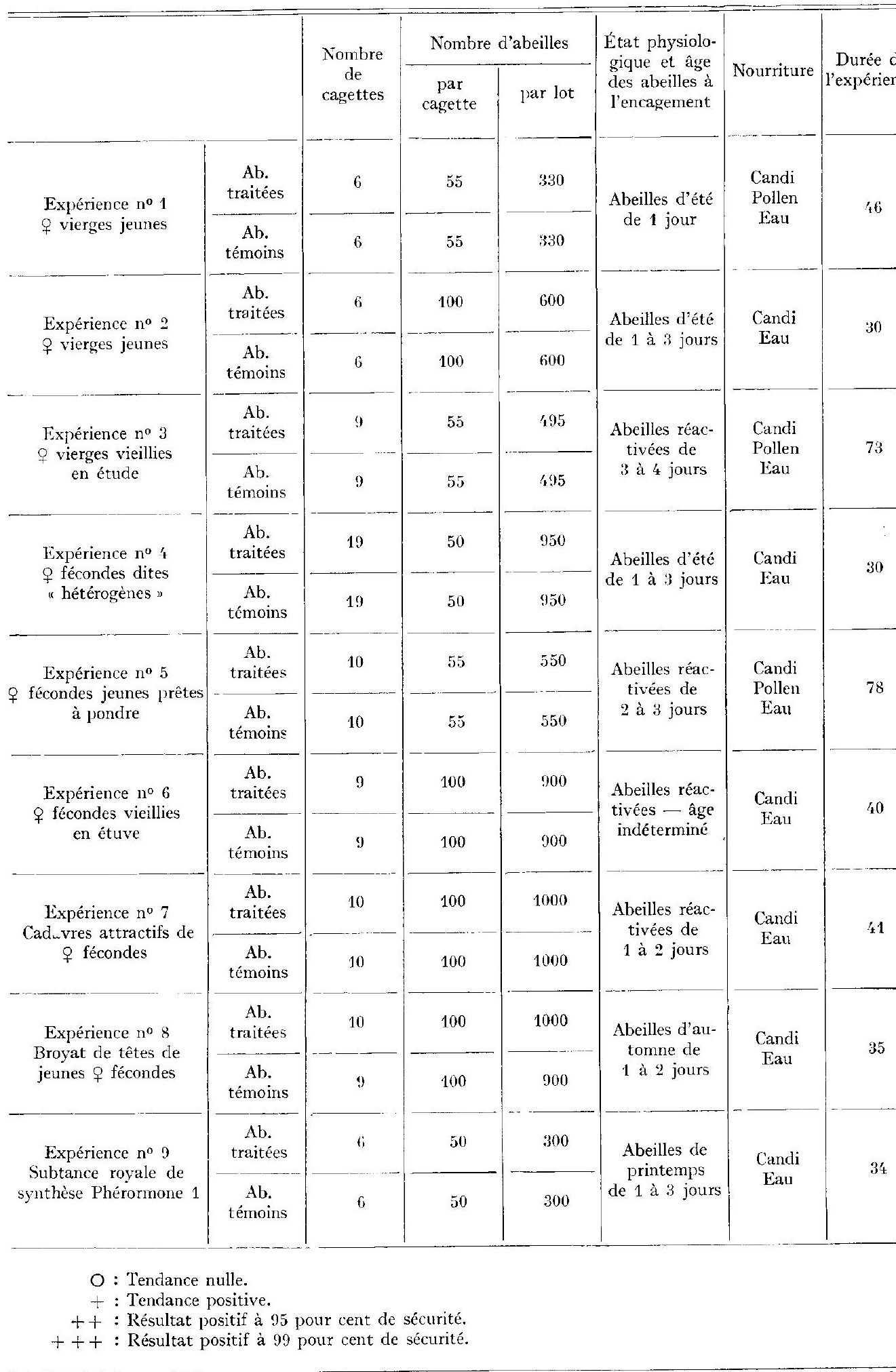


Différence maximum de mortalité entre les 2 lots $\%$

\begin{tabular}{|c|c|c|c|c|c|c|}
\hline \multicolumn{2}{|c|}{$\%$} & \multicolumn{2}{|c|}{$\%$} & \multirow{2}{*}{$\begin{array}{c}\begin{array}{c}\text { Sur la 1ere partie } \\
\text { de l'expérience }\end{array} \\
+ \\
+ \text { du } 1^{\text {er }} \text { au } 23^{\mathrm{e}} \mathrm{j}\end{array}$} & \multirow{2}{*}{$\begin{array}{l}\begin{array}{c}\text { Sur la 2e partie } \\
\text { de l'expérience }\end{array} \\
+ \\
\begin{array}{c}\text { du } 2 \mathrm{e}^{\mathrm{e}} \text { au } 46^{\mathrm{e}} \mathrm{j}\end{array}\end{array}$} & \multirow{2}{*}{$\begin{array}{c}\begin{array}{c}\text { Sur l'ensemble } \\
\text { de l'expérience }\end{array} \\
+ \\
\text { du } 1^{\text {er }} \text { au } 46^{\circ}\end{array}$} \\
\hline $10 \mathrm{ab}$. & 12 & $33 \mathrm{ab}$. & 10 & & & \\
\hline $200 \mathrm{ab}$. & 33 & 173 ab. & 28 & du $1^{\text {er }} \stackrel{+}{\text { aul }} 17^{\mathrm{e}} \mathrm{j}$ & du $18^{+\mathrm{e}}$ au $26^{\mathrm{e}} \mathrm{j}$ & $\underset{\text { du } 4^{\text {er }} \text { au } 30^{\circ} j}{ }$ \\
\hline $123 a b$. & 25 & $25 \mathrm{ab}$. & 5 & du $\mathbf{1}^{+\mathrm{er}}$ aut $\mathbf{k}^{\prime} \mathbf{y}^{\mathrm{e}} \mathrm{j}$ & $\mathrm{du} 45^{\mathrm{e}}$ at $73^{\mathrm{e}} \mathrm{j}$ & du $\mathrm{f}^{\mathrm{er}}$ aut $73^{\mathrm{e}} \mathrm{j}$ \\
\hline 81 ab. & 8,5 & $65 \mathrm{ab}$. & 7 & $\stackrel{O}{\text { du } 1^{\text {er }} \text { aul } 15 \mathrm{e}^{\mathrm{e}} \mathrm{j}}$ & $\underset{\mathrm{du} 16^{\mathrm{e}} \text { an } 30^{\mathrm{e}} \mathrm{j}}{ }$ & 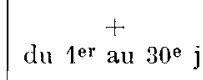 \\
\hline 8.5 ab. & 12 & $17 \mathrm{ab}$. & 3 & du $\stackrel{+}{\text { er }}$ au $39^{e} \mathrm{j}$ & $\stackrel{+}{\mathrm{du}} 40^{\mathrm{e}}$ au $78^{\mathrm{e}} \mathrm{j}$ & $\stackrel{+}{\text { du } 1^{\text {er }} \text { au } 78^{\mathrm{e}} \mathrm{j}}$ \\
\hline 110 ab. & 12 & $110 \mathrm{ab}$. & 12 & $\stackrel{O}{\text { du } 1^{\text {er }}}$ au $18^{e} j$ & du $19^{\mathrm{e}}$ au $40^{\mathrm{e}} \mathrm{j}$ & du ${ }^{\text {er }} \stackrel{+}{\text { au }} 40^{\mathrm{e}} \mathrm{j}$ \\
\hline 185 ab. & 18,5 & $185 \mathrm{ab}$. & 18,5 & $\stackrel{O}{\mathrm{du} 1^{\text {er }} \text { an } 18^{\mathrm{e}} \mathrm{j}}$ & du $19^{+\frac{1}{t}}$ au $4^{1} 1^{\mathrm{e}} \mathrm{j}$ & du $1^{\text {er }}$ au $41^{\mathrm{e}} \mathrm{j}$ \\
\hline $121 \mathrm{ab}$. & 12 & $51 \mathrm{ab}$. & 5 & $\stackrel{O}{\text { du } 1^{\text {er }} \text { au } 17^{-} j}$ & du $18^{+}$au $35^{e} j$ & $\underset{+}{+}$ \\
\hline $8 \mathrm{ab}$. & 2 & () ab. & 0 & $\stackrel{O}{\mathrm{du} 1^{\mathrm{er}}} \stackrel{\mathrm{au} 17^{\mathrm{e}} \mathrm{j}}{ }$ & $\stackrel{O}{\text { du } 18^{\mathrm{e}} \text { au } 3^{\prime} \mathbf{e}^{\mathrm{e}} \mathrm{j}}$ & $\stackrel{O}{\text { du } 4^{\text {er }} \text { au } 34^{\mathrm{e}} j}$ \\
\hline
\end{tabular}

Différence de mortalité en fin d'expérience $\%$ Analyse statistique des courbes 
I,e test $F$ de signification est donné par la relation :

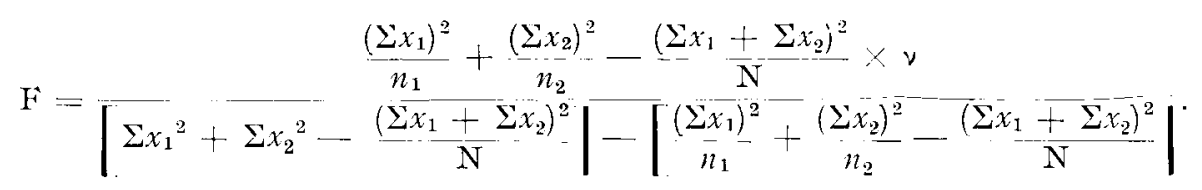

Pour l'interprétation des résultats, les calculs ont porté non seulement sur la durée totale de l'expérience mais aussi sur des fractions plus ou moins longues de cette durée, fixées selon la forme des deux courbes.

Les pentes des courbes de mortalités indiquent, à notre avis, l'effet plus ou moins perceptible de la reine sur le taux de mortalité des ouvrières accompagnatrices.

Le tableau I indique les résultats de ces analyses. Il montre que sur l'ensemble des 9 expériences, 3 sont statistiquement valables. Les 6 autres montrent à un certain moment une tendance positive de la part de la reine à exercer une action favorable en réduisant le taux de mortalité des ouvrières.

Expérience $n^{\circ} 1$.

Nous obtenons une valeur de $\mathrm{F}$ non significative pour la durée totale de l'essai. Cette valeur reste d'ailleurs la même sur les différentes portions ou parties de courbes analysées.

Expérience $n^{0} 2$.

La valeur de F n'est pas significative pour la durée totale de l'expérience. Les différentes analyses portant sur plusieurs parties des courbes de mortalité, indiquent $\mathrm{du} \mathrm{I}^{\mathrm{er}}$ au $I 7^{\mathrm{e}}$ jour, une valeur de $\mathrm{F}$ non significative, mais du I $8^{\mathrm{e}}$ au $26^{\mathrm{e}}$ jour, cette valeur devient significative. Elle ne l'est plus du $18^{\mathrm{e}}$ au $3 \mathrm{O}^{\mathrm{e}}$ jour, au seuil de $5 \mathrm{p}$. Ioo.

Expérience $n^{\circ} 3$.

Ia valeur de F n'est pas significative pour la durée totale de l'expérience, mais cette valeur est significative au seuil de I p. Ioo du I er au $44^{\mathrm{e}}$ jour, c'est-à-dire pour une période dépassant largement la moitié de la durée totale de l'expérience : l'écart entre les 2 courbes augmente. I u $45^{\mathrm{e}}$ au $73^{\mathrm{e}}$ jour, la valeur de $\mathrm{F}$ n'est plus significative : l'écart entre les 2 courbes diminue.

Expérience $n^{\circ} 4$.

La valeur de $\mathrm{F}$ n'est pas significative pour toute la durée de l'expérience ni pour la I $^{\mathrm{re}}$ ou la $2^{\mathrm{e}}$ partie de celle-ci.

Expérience no $n^{\circ}$.

I a valeur de $F$ n'est pas significative pour l'ensemble de la courbe, comme pour les parties de celle-ci comprises entre le $\mathrm{I}^{\mathrm{er}}$ et le $39^{\mathrm{*}}$ jour et entre le $40^{\mathrm{e}}$ et le $78^{\mathrm{e}}$ jour.

\section{Expérience $n^{\circ} 6$.}

Les résultats pour l'ensemble de la courbe donnent une valeur de $F$ non significative. Les valeurs de $\mathrm{F}$ sont les mêmes du $\mathrm{I}^{\mathrm{er}}$ au $\mathrm{I} 8^{\mathrm{e}}$ jour et du $\mathrm{I}_{9}^{\mathrm{e}}$ au $4^{\mathrm{e}}$ jour, bien que les courbes s'écartent dans la $2^{\mathrm{e}}$ partie de l'essai. 
Expérience no $\%$.

La valeur de F' n'est pas significative pour la durée totale de l'expérience ainsi que pour le début de celle-ci, soit du I er au $I 8^{\mathrm{e}}$ jour. Mais à partir du I $\mathrm{g}^{\mathrm{e}}$ jour jusqu'au $4 \mathrm{I}^{\mathrm{e}}$, la valeur de $\mathrm{F}$ est significative au seuil de $5 \mathrm{p}$. Ioo.

Expérience $n^{\circ} 8$.

La valeur de li n'est à aucun moment significative.

Expérience $n^{\circ} 9$.

La valeur de $\mathrm{F}$ est identique à celle de l'expérience précédente. Elle n'est pas significative.

\section{DISCUUSSION}

Les résultats de l'analyse statistique au moyen clu test $\mathrm{F}$ n'expliquent pas la position systématique des 2 courbes de mortalité l'une par rapport à l'autre et la situation toujours plus favorable de la courbe des abeilles traitées comparée aux témoins. Tout au long des expériences et même lorsque les coutbes sont très rapprochées (expér. nos 8 et 9), nous obtenons che $\%$ les témoins des mortalités toujours supérieures à celles des traitées.

Nous pensons done que le calcul statistique utilisé pour l'interprétation de ces expériences ne met en évidence l'influence des reines que lorsque celle-ci est particulièrement forte comme c'est le cas pour les reines vierges vieillies en étuve. Quand cette influence se fait sentir à un moindre degré, l'analyse statistique est sans doute trop sévère ou non appropriée et donne toujours des résultats non significatifs alors que dans tous les cas sans exception, la position des courbes de mortalité montre qu'il existe une influence de la reine.

C'est pourquoi, en vue de permettre un autre type d'interprétation des résultats, nous avons entrepris de présenter l'évolution des phénomènes sous une forme différente.

Nous exprimons cette fois les résultats, non plus en mortalité, mais en jours de vie. I) I $^{\text {er }}$ au dernier jour de l'expérience, nous avons évalué pour chaque cagette prise dans un lot, le nombre d'abeilles restant en vie. Sur la durée totale de l'expérience, nous obtenons la somme des jours de vie d'abeilles dans une cagette. En divisant cette somme par le nombre d'abeilles introduites dans une cagette au début de l'expérience, nous calculons la durée moyenne de vie d'une abeille dans une cagette d'un des lots. Nous avons calculé ensuite la durée moyenne de vie d'une abeille à l'intérieur d'un lot, en effectuant la somme des durées moyennes de vie d'une abeille dans chaque cagette et en la divisant par le nombre de cagettes constituant un lot (fig. Io).

Sur cette figure, nous indiquons les différentes durées moyennes de vie obtenues pour une abeille dans chaque cage et dans chacun des lots.

Les différences de durées moyennes de vie entre les 2 lots indiquent l'influence de la reine sur la durée moyenne de vie d'une ouvrière.

Analysons maintenant les résultats obtenus par cette méthode : 

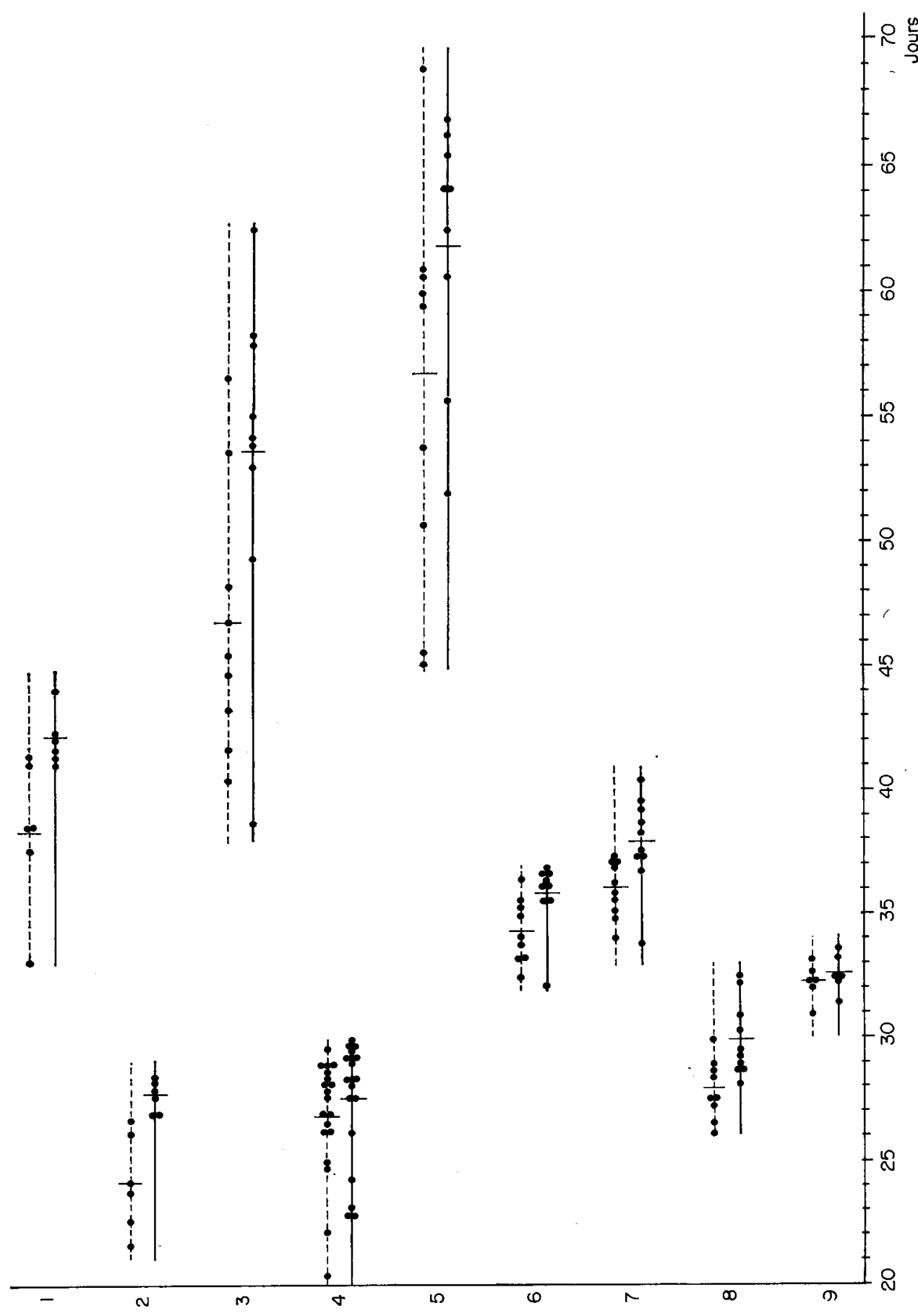
Les jeunes reines vierges accompagnées d'ouvrières nourries de pollen augmentent de près de 4 jours la durée moyenne de vie d'une ouvrière. L'augmentation de la durée moyenne de vie est à peu près du même ordre lorsque les ouvrières sont privées de pollen. Ceci paraît indiquer que l'influence des reines vierges se manifeste avec la même intensité, en présence ou en 1'absence de l'aliment azoté. L'étude des courbes de mortalité montre qu'en l'absence de pollen, le nombre total d'abeilles mortes est plus élevé et l'analyse statistique donne un résultat significatif. Elle n'en donne pas lorsqu'en présence de ces mêmes reines, on donne du pollen aux ouvrières. L'étude des durées moyennes de vie montre cependant que, dans les 2 cas, les reines ont exercé une action similaire.

Quelle que soit la méthode d'interprétation utilisée, ce sont les reines vierges vieillies en étuve qui augmentent le plus la durée moyenne de vie des ouvrières car nous trouvons une différence de près de 7 jours entre les 2 lots.

Les reines fécondes hétérogènes n'augmentent pratiquement pas la durée moyenne de vie tandis que les jeunes reines fécondes dont les ouvrières reçoivent du pollen l'augmentent de plus de 5 jours. Les jeunes reines fécondes exercent une influence plus forte sur la durée moyenne de vie des ouvrières que les jeunes reines vierges ne recevant pas de pollen. Ces dernières ont cependant une action significative sur la mortalité.

Les reines fécondes vieillies en étuve prolongent d'un jour et demi la durée moyenne de vie des ouvrières. Elles manifestent donc encore une action sur la prolongation de la vie des abeilles plus importante que celle des reines fécondes dites hétérogènes mais beaucoup moins importante que celle des reines fécondes jeunes. L'analyse statistique des mortalités en présence de reines fécondes jeunes ou vieilles ne donne pas de résultats significatifs, cependant nous notons une différence de 3 jours et demi de durée moyenne de vie entre ces deux types de reines.

Les cadavres de reines augmentent de près de 2 jours la durée moyenne de vie des ouvrières et les broyats de têtes de jeunes reines ont une action de même intensité. Les analyses statistiques positives dans le I $^{\mathrm{er}}$ cas indiquent une influence réelle de la part des reines mortes. Les analyses statistiques non significatives dans le $2^{\mathrm{e}}$ cas montrent que les broyats céphaliques n'ont eu aucun effet statistiquement prouvé bien qu'ils aient aussi réduit les mortalités. Les durées moyennes de vie comparables font supposer cependant que les broyats se sont montrés aussi actifs que les reines mortes entières.

L'effet de la phérormone I est presque inexistant. La différence entre abeilles traitées et abeilles témoins est très faible mais reste en faveur des traitées. Ici les résultats non significatifs de l'analyse statistique concordent bien avec ceux obtenus par l'évaluation des durées moyennes de vie.

L,es deux méthodes en se complétant permettent de mieux comparer les différentes reines utilisées.

\section{CONCLUSION}

L,es résultats qui viennent d'être exposés nous amènent à conclure de la façon suivante :

Le test $F$ effectué sur l'ensemble des mortalités ne s'est jamais révélé statis- 
tiquement significatif si l'on considère la durée totale cles expériences. Cependant, d'après les courbes, les reines exerceraient leur action au bout d'un temps variable compris entre 18 et 26 jours (exp. $n^{\circ} 2$ ), entre 27 et 44 jours (exp. $n^{0} 3$ ), entre I9 et $4 \mathrm{I}$ jours (exp. $\mathrm{n}^{\circ} 7$ ). L'analyse statistique donne alors des résultats positifs sur une portion de la courbe.

D'ailleurs, les différences maxima des mortalités sont toujours situées dans la deuxième partie de l'essai, jamais avant le $26 \mathrm{e}$ jour (exp. $n^{\circ} 2$ ) ou le $28^{\mathrm{e}}$ jour (exp. $n^{\circ} 8$ ). Dans l'expérience $n^{\circ} 7$, cette différence maximutm apparaît assez tardivement (le $4 \mathrm{I}^{\circ}$ jour) pour donner une valeur de $\mathrm{F}$ significative.

1)'après 1'aspect des courbes et leur pente, les mortalités des abeilles d'èté ne recevant pas de pollen, apparaissent plus tôt que celles des abeilles réactivées, nourries dans les mêmes conditions. Dans le premier cas, elles apparaissent vers le $\mathrm{I} 7^{\mathrm{e}}$ et le

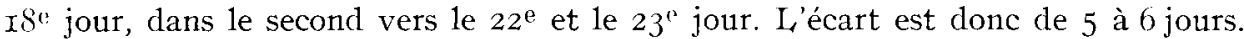

Jès que les abeilles reçoivent du pollen, les mortalités commencent à peu près en même temps chez les abeilles jeunes d'été et chez celles qui proviennent de ruches chauffées.

En ce qui concerne l'action du pollen, nous confirmons les données acquises par les auteurs précédemment cités. Si nous comparons les courbes de mortalité des expériences $n^{\circ} \mathrm{I}$ et 2 , celles de l'expérience $n^{\circ}$ I ne s'écartent de l'axe des abscisses qu'asse $z$ tard. Celles de l'expérience $n^{\circ} 2$ s'éloignent rapidement de cet axe. Mais l'apport de pollen n'empêche pas les reines de réduire encore le taux de mortalité des ouvrières, comme on peut le constater dans $1^{\prime}$ expérience $\mathrm{n}^{\circ} 5$ et surtout dans l'expérience $\mathrm{n}^{\mathrm{o}} 3$ qui s'échelonnent sur plus de 70 jours. Si la présence de pollen retarde et diminue effectivement les mortalités, elle n'empêche pas les reines de manifester leur influence sur celles-ci.

Il ressort du tableau I, des courbes de mortalité (fig. I à g) et des durées moyennes de vie (fig. Io) que ce sont les reines vierges vieillies à l'étuve qui produisent l'effet maximum.

En rapport avec des données déjà acquises (Butler, I960; PAIN, I959, I96I), en supposant que la quantité de produits volatils et celle de phérormone I vont de pair pour la détermination de l'odeur attractive, nous avançons l'hypothèse que les reines vierges vieillies seraient susceptibles de contenir dans leurs glandes mandibulaires plus de substances que les reines vierges jeunes.

Il est tout à fait nouveau de signaler que des cadavres de reines fécondes attractives (exp. $n^{0} 7$ ) produisent une action de même intensité bien que plus tardive que celle des jeunes reines vierges (exp. $n^{\circ} 2$ ), en réduisant significativement le taux de mortalité des ouvrières accompagnatrices. Cependant, d'après l'étude des durées moyennes de vie, leur influence est un peu moins marquée que celle des reines vierges vivantes. L'action des broyats céphaliques attractifs corrobore en partie ces résultats. L'odeur attractive des cadavres ou des broyats céphaliques a probablement joué un róle puisque la présence de cadavres de reines vierges naissantes non attractives est sans action sur les mortalités.

I)'autre part, tenant compte de certaines observations, nous nous sommes demandé si l'élevage de couvain par les ouvrières captives et l'activité d'étirage des cires pouvaient modifier les mortalités. Pour ne citer que quelques cas :

- dans l'expérience $n^{0} 2$ qui est statistiquement significative, il y a eu étirage 
de la cire et ponte par la reine vierge chez les abeilles traitées et pas de ponte par les ouvrières pondeuses chez les abeilles témoins ;

- dans l'expérience $n^{\circ} 3$, également statistiquement significative, il y a eu étirage de la cire, ponte par la reine et élevage chez les abeilles traitées. Chez les témoins, il y a eu ponte par ouvrières pondeuses et élevage mais l'étirage de la cire a été plus tardif chez ces derniers; dans l'expérience $\mathrm{n}^{\mathbf{0}} \mathbf{5}$, non significative, des observations identiques ont été faites ;

- dans l'expérience $n^{\circ} 7$ seulement, un étirage partiel de la cire a été noté dans les deux lots.

Ces observations non systématiques n'apportent à notre avis aucun élément susceptible de modifier nos conclusions.

Par contre, le comportement des ouvrières en présence de reine nous a paru tout à fait caractéristique. Dans tous les cas, les abeilles, sauf pour l'expérience $\mathrm{n}^{0} 6$, se sont maintenues très calmes auprès de leurr reine ; celle-ci paraît agir sur elles en les tranquillisant pendant plus de la moitié de la durée des expériences.

Au contraire, les abeilles témoins, surtout lorsqu'elles sont maintenues sur du candi sans pollen, manifestent une grande agitation.

Signalons encore que les cagettes contenant des abeilles avec reine restent bien plus longtemps exemptes de déjection que celles des abeilles témoins. L,es ouvrières accompagnées d'une reine défèquent beaucoup plus tard que les ouvrières orphelines.

Pour conclure, nous dirons que, dans toutes les expériences sans aucune exception, les courbes représentant les mortalités des ouvrières en présence de reines, vivantes ou mortes, de leur extrait naturel ou du produit de synthèse (phérormone I) se situent toujours au-dessous de celles des ouvrières orphelines. L'influence des reines s'exerce indépendamment de la présence du pollen.

Le fait que la phérormone I non attractive n'ait que peu d'action, fait supposer que l'on se trouve en présence d'un phénomène qui n'est pas en relation avec cette substance, mais peut-être avec d'autres produits de nature plus ou moins phérormonale. L'action de stimuli visuels, tactiles ou autres peut être discutée mais semble cependant fort peu probable compte tenu des résultats enregistrés dans les expériences ne faisant pas intervenir les reines mortes.

Reçu pour publication en novembre 1965.

\section{SUMMARY}

THE INFLUENCE OF THE QUEEN BEF ("APIS MELLIFICA L. ") ON THE MORTALITY RATE OF THE ATTENDANT WORKERS

In the course of a research on the interactions between queen and worker bees, the authors studied the way in which the mortality of attendant workers was affected by the presence of the following : live queens, virgin or fecund, young or old ; dead queens ; ground-up queen's heads; synthetic pherormone $\mathrm{I}$.

Analysis of the mortality curves shows that the presence of live queens lowers the mortality rate of small groups of 50 to too workers but to varying degrees. It is the virgin queens aged in incubators which reduce this mortality most. The presence of pollen does not conceal the influence of the queens.

Annales de l'Abcille. - I 966. 
The corpses of attractive fertile queens have the same effect on the reduction of the mortality rate. This effect is of the same intensity as that of the live young virgin queens.

The influence of the queens is only felt after the workers have been encaged with the queens for more than $I_{5}$ days.

Non-attractive, synthetic pherormone I only slightly reduces the mortality rate of the workers. The hypothesis of a pherormonal effect is put forward, linked perhaps with the smell of the queen, but other factors could also come into play.

\section{RÉFÉRENCES BIBLIOGRAPHIQUES}

Anderson J., 193. How long does a bee live? Bee World, 12, 25.

BACK E., 1956. Finfluss der im Pollen enthaltenen Vitamine auf Lebensdauer, Ausbildung der Pharynxdrüsen und Brutfähigkeit der Honigbiene. Insectes sociaux, 3, 285-292.

Barbier M., Lederer E., I960. Structure chimique de la "substance royale " de la reine d'abeille (Apis mellifica). C. R. Acad. Sci., 250, 4467-4469.

BERTHOLF L. M., I942. Effect of certain biological factors on the longevity of caged bees. J. econ. Entomol., 35 (6), 887-89I.

Beutler R., Opfinger E., I950. Pollenernährung und Nosemabefall der Honigbiene (Apis mellifica). Z. vergleich. Physiol., 32, 383-42I.

ButLer C. G., Ig60. Queen substance production by virgin queen honey-bees. Proc. r. entomol. Soc. London, 35, 10-12.

ButLeR C. G., I96I. The scent of queen honeybees (A. mellifera L.) that causes partial inhibition of queen rearing. J. Insect. Physiol, $7,258-264$.

Butler C. G., Paton P. N., ig62. Inhibition of queen rearing by queen honeybees (Apis mellifera L.) of different ages. Proc. $r$. enlomol. Soc. London A, 37, 7-9.

Buttel-ReEpen H. V., 1915. Leben und Wesen der Bienen, Braunschweig.

Chalvin R., 1952. Sur le déterminisme de l'effet de groupe chez les abeilles. Physiol. comp. OEcol., 282-287.

Chauvin R., Darchen R., Pain J., I96r. Sur l'existence d'une hormone de construction chez les abeilles. C. R. Acad. Sci., 253, II $35^{-1} 136$.

Demuth G. S., 1926. Duration of life of the worker bee. Glean. Bee cullure, 54, 106.

EL-DEEB A. L. A., 1952. Longevity of some races of the honeybee (Apis mellifera L.). Abstract of a thesis. Univers. of Illinois. Urbana III, 92 (12), 517.

Evenius Ch., I937. Beobachtungen an der Schlunddrüse der Honigbiene während der Winterruhe. Dtsch. Imkerführer, 128 .

FARRAR C. L., 1936. Influence of pollen reserves on the surviving population of overwintered colonies. Amer. Bee J., r $7 \mathbf{6}(9), 45^{2-455}$.

FreE J. B., SPENCER-Booth Y., 1959. The longevity of worker honeybees (Apis mellifera). Proc. $r$. entomol. Soc. London A, 34 (10/12), I41-150.

Free J. B., Spencer-Booth Y., Ig60. Chill-coma and cold death temperatures of Apis mellifera. Entomol. exper. appl., 3 (3), 222-230.

FreE J. 13., SPENCER-Bootu Y., 1962. The upper lethal temperatures of honeybees. Entomol. exper. appl., Amsterdam, 5, 249-254.

Fresnaye J., 1965. La durée de vie des reines d'abeilles en cages d'expédition. Ann. Abeille, 8, 95-107.

Giordani G., 1957. Yeast in the feeding of honeybees. Bee World, 9-58, 243. Apicollore d'Italia, 24 (5-6), $125^{-160 .}$

Govtarski H., I950. Die Bedeutung der Nährhefe als Pollenersatz. Südwestd. Imker., 2 (2), 28-29.

Gontarski H., I 954. Untersuchungen über die Verwertung von Pollen und Hefe zur Brutpflege der Honigbiene. Z. Bienenforsch., 2, 5 Jahrg. 6.

Grasse P. P. et Chauvin R., ig44. L'effet de groupe et la survie des neutres dans les sociétés d'insectes. Rev. Scient., 7, 461-464.

DE Groot A. P., I95I. Leffect of protein containing diet on the longevity of caged bees. Kon. Ned. Akad. Welenschappen. Proceedings, Série C, 54, $27^{2}$.

DE Groot A. P., 1953. Protein and amino acid requirements of the honeybee (A. M. I..). Physiol. comp. Ecol., 3, 197-285.

Hassanein M. H., EL-Banby M. A., 1960. Studies on the longevity of Carniolan, Caucasian and Italian honeybee workers, with special reference to their foraging behaviour. Bull. Soc. Entomol. Egypte, 44, $291-308$.

HAYDAK M. H., 1937. The influence of a pure carbohydrate diet on newly emerged honeybees. Ann. Entomol. Soc. Amer., 30, 258-262. 
Unjtuanek J., I933. Studies on the feeding of the honeybee with pollen (Apis mellifica L.). Cesky vcelar, 67 ( 10$), 379-385$.

Hejtmanek J., 1943. Die Pollenverdaung der Honigrbiene. Prievidja.

Hüsing J. O., 1952-I953. Beobachtungen über die Lebensdauer von Bienenarbeiterimnen. Wissensch. Z. Marin-Iulher Uniw. Halle-Wiltenberg., Jahrg. II, 11, 779-785.

KAPIL R. P'., I957. The length of life and the brood-rearing cycle of the Indian bee. Bee World, 38, $25^{8-263 .}$

Keld.er G., 1920. Wie alt werden die Bienen? Arch. Bienenkde. 2, i 80.

Kelder-Kitzinger R., I935. Kam die erwachsene Arbeiterin der Honigbiene (Apis mellifica) Eiweiss verwertell? Z. vergleich. Physiol., 22, г-3.

Kofhler A., т92I. Weist die Biene in ihrem körper Reserverstoffe für die Winterruhe auf? Schweiz. Bienenlsg., 44, 424-42S.

KRATKY E.., I93I. Morphologie und Physiologrie der Drüsen in Kopf und Thorax der Honigbiene (A. M. L.) • Z. Wissens. Zool., 139 (1), i $20-200$.

Lotmar R., I939. Sojamehl und Futtersaftdrüsen. Schreeiz. Bienenslg., 62, 339-340.

Louveaux J., 1958. Recherches sur la récolle dlu pollen par les abeilles (Apis mellifica L.). Thèse Sci. Nat. Paris, $\mathrm{H}^{\circ} 3997$.

MnUekmayer G., r954. Untersuchungren über die Beziehungen zwischen arbeitsleistung und Lebensdauer bei Arbeiterimen der IIonigbiene. Arch. Bienenkde, 31 (I), 31-4I.

MaURizio A., 1946. I3eobachtungen über die Lebensdauer und den Futterverbrauch gefangen gehaltener Bienen. Beih. Schaeiz. Bienenztg., 2 (г3), I-t+.

Mavrizio A., I954. Pollenernahrungr und Lebensvorgänge bei der Ilonigbiene. (Apis mellifica L.). Landw. Jb. Schtoeiz., 68 (2), I $15^{-\mathrm{J}} 82$.

Maurizio A., i959. Factors influencing the lifespan of bees. Ciba Foundation Symposium on the Lifespan of Animals, $23 \mathrm{I}-243$.

Melampy R. M., MCGregor S. İ., I 39 . Nutritional value of certain foods for the adult honeybee. J. econ. Entomol., 32, 721-725.

Milum V. (i., I931. The honeybee's span of life. Thirlieth Ann. Rep. Illinois Stare Beekeepers' Assoc., 94-107.

Moskovi.jevic V., 1939. Eine neue Auffassung vom Gesellschaftsleben der Ilonigbiene. Arbeiten des 12. Int. J3ienenzuchtkongress. Zürich, I939. Schweiz. Bienenzlg., 689 (Bee World.), 21, 39.

Mussbiciler A., i952. Die Bedeutung aüsserer Einflüsse und der Corpora allata bei der Afterweiselentstehung von Apis mellifica. Z. vergleich. Physiol., 34, 207-221.

Nickel H. K., Arubruster L., ig37-1938. Vom Lebenslauf der Arbeitsbiene. Arch. Bienenkde, 18, 257,19, г 34 .

Pain J., I954. Sur l'ectohomone des reines d'Abeilles. C. R. Acal. Sci., Paris, 239, I869-1870.

Pain J., i955. Influence des reines mortes sur le développement ovarien de jeunes ouvrières d'Abeilles (Apis mellifica). Insectes sociaux, 2 (1), 35-43.

Paiv J., 1959. Étude de l'apparition de l'attractivité chez les reines vierges d'abeille. C. R. Acad. Sci., Paris, 248, 32 I $1-32$ I2.

Pain J., I96r. Sur la phérormone des reines d'abeilles et ses effets physiologiques. Thèse Sc. Nat., Paris, $\mathrm{n}^{\mathrm{O}}+526$.

PAIN J., ig6r. Sur quelques facteurs alimentaires accélérateurs du développement des oufs dans les ovaires des ouvrières d'abeilles (.Apis mellifica I..). Insectes Socianx, 8 (1), 3I-93.

Peterka V., Svoboda J., 1937. "Sojasan "als Pollenersatzmittel. Dtsch. Imker 3, 1939, Cesky Vcelar, 72, 9 I-96 en tchèque.

Peterka V., 1939. Sojamehl und die Frage der Pollenersatzmittel. Schweis. Bienenstg, 62 (3), I43.

Phillips F. F., 1922. The effect of activity of the length of life of honeybees. J. econ. Entomol., 15, 368.

Phillips I. F., I928. Beekecping, New-York.

Phillırs li. F., 1939. Beekeeping. Macmillan, New-York.

Phillips IE. I., i946. Temperature and length of life of bees. Bee World., 9 (27), $73-74$.

Pixelit-Goodricil II. I. M., I920. Determination of age in honey-bees. Quart. J. microsc. Sci., 64 .

Riley C. V., 1895. Jongevity in insects. Proc. Entomol. Soc. Washington, 3, I08-125.

Rocksteiv II., 1950. Longevity in the adult worker honeybee. Ann. Enlomol. Soc. Amer., 43, I52-154.

Röscir G. A., 1925. Lintersuchungen über die Arbeitsteilung im Bienenstaat. Z. vergleich. Physiol., $57 \mathrm{I}-63 \mathrm{I}$.

Schmidt H., I923. L̀ber den Alterstod der Biene. Z. Nalurforsch., 49.

SENN C., 192r. Das Alter der Arbeitsbienc. Schweiz. Bienenzlg. 307.

Soubek St., 1927. The pharyngeal glands of the honeybee. Bull. Ec. sup. Agron. Brno. C., 10, I-63. Svoboda J., 19 o. Ủber den Wert des Polleus als Nahrungsmittel für Bienen. Schweiz. Bienenzig., 63, 206-209. 
VoogD C., 1959. How worker bees perccive the presence of their queen. Z. vergleich. Pliysiol., 41, 527-582 Wail $0 ., 195^{6}$. Comparative studies on the nutritive value of different types of pollen and pollen substitutes. Riassunti XVI Congr. Int. Apic. Vienna, 92.

WaIIL O., I963. Vergleichende Untersuchungen über den Nährwert von Pollen; IIefe, Sojamehl und Trockenmilch für die Honigbiene. Z. Bienenforsch., 6 (8), 209-280.

WoODrow A. W., i935. Some effects of relative humidity on the length of life and food consumption of honeybees. J. econ. Entomol., 28, 565-568.

Woodrow A. W., I94I. Some effects of temperature, relative humidity, confinement and type of tood on queen bees in mailing cages. U.S. Dept. agric. Bur. Enton. Quar., E529, 13. 